\title{
Lobectomy versus stereotactic body radiotherapy in healthy patients with stage I lung cancer
}

\author{
Joshua E. Rosen, BASc, ${ }^{a}$ Michelle C. Salazar, MD, ${ }^{a}$ Zuoheng Wang, PhD, ${ }^{b}$ James B. Yu, MD, MHS, \\ Roy H. Decker, MD, PhD, ${ }^{\mathrm{c}}$ Anthony W. Kim, MD, ${ }^{\mathrm{a}}$ Frank C. Detterbeck, MD, ${ }^{\mathrm{a}}$ and Daniel J. Boffa, MD ${ }^{\mathrm{a}}$
}

\begin{abstract}
Objectives: Stereotactic body radiotherapy is an effective treatment for patients with early-stage non-small cell lung cancer who are not healthy enough to undergo surgery; however, the relative efficacy versus surgery in healthy patients is unknown. The National Cancer Database contains information on patient health and eligibility for surgery, allowing the long-term survival associated with lobectomy and stereotactic body radiotherapy to be compared in healthy patients with clinical stage I disease.
\end{abstract}

Methods: The National Cancer Database was queried for patients who underwent lobectomy or stereotactic body radiotherapy for clinical stage I lung cancer between 2008 and 2012. Healthy patients were selected by excluding patients not offered surgery because of health-related reasons and only including patients documented to be free of comorbidities.

Results: A total of 13,562 comorbidity-free patients with clinical stage I lung cancer treated with lobectomy were compared with 1781 patients treated with stereotactic body radiotherapy. Time-stratified Cox proportional hazards models found lobectomy to be associated with a significantly better outcome than stereotactic body radiotherapy for both T1N0M0 tumors (hazard ratio, 0.38; $95 \%$ confidence interval, $0.33-0.43 ; P<.001)$ and T2N0M0 tumors $5 \mathrm{~cm}$ or less (hazard ratio, 0.38; confidence interval, 0.31-0.46; $P<.001$ ). In a propensity-matched analysis of 1781 pairs, lobectomy remained superior to stereotactic body radiotherapy $(5$-year survival $59 \%$ vs $29 \%, P<.001)$. Furthermore, when the subset of stereotactic patients who had refused a recommended surgery $(\mathrm{n}=229)$ were propensity matched to lobectomy patients, lobectomy was associated with improved survival (5-year survival $58 \%$ vs $40 \%, P=.010$ ).

Conclusions: Among healthy patients with clinical stage I non-small cell lung cancer in the National Cancer Database, lobectomy is associated with a significantly better outcome than stereotactic body radiotherapy. Further study is warranted to clarify the comparative effectiveness of surgery and stereotactic body radiotherapy across various strata of patient health. (J Thorac Cardiovasc Surg 2016;152:44-54)

Lung cancer is responsible for more than 150,000 deaths each year in the United States, representing the nation's leading cause of cancer-related mortality. ${ }^{1}$ Historically,

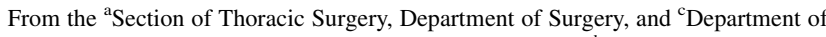
Therapeutic Radiology, Yale School of Medicine; and ${ }^{b}$ Yale School of Public Health, New Haven, Conn.

Received for publication Nov 17, 2015; revisions received March 4, 2016; accepted for publication March 13, 2016; available ahead of print April 27, 2016.

Address for reprints: Daniel J. Boffa, MD, 330 Cedar St, BB205 PO Box 208062,

New Haven, CT 06520-8062 (E-mail: Daniel.boffa@yale.edu).

$0022-5223 / \$ 36.00$

Copyright (c) 2016 by The American Association for Thoracic Surgery

http://dx.doi.org/10.1016/j.jtcvs.2016.03.060

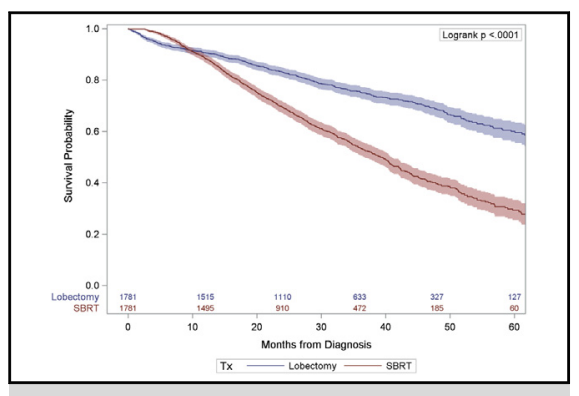

Overall survival of matched patients with stage I NSCLC treated with lobectomy or SBRT.

\section{Central Message}

Lobectomy is associated with superior 5-year survival versus SBRT among healthy patients with clinical stage I NSCLC in the NCDB.

\section{Perspective}

The tendency to treat medically inoperable patients with SBRT creates a health-related bias against it that may compromise retrospective comparisons of SBRT with lobectomy in large databases. By focusing on healthy patients, the present study minimizes health-related bias against SBRT and shows lobectomy to be associated with a superior 5-year survival over SBRT in healthy patients with stage I NSCLC.

See Editorial page 1. the best outcomes for early-stage lung cancer have been achieved through anatomic resection of the primary tumor via lobectomy. ${ }^{2}$ However, lobectomy is a major surgical procedure, and a significant proportion of patients with early-stage lung cancer are not sufficiently healthy to

Scanning this QR code will take you to supplemental figures and tables for this article.

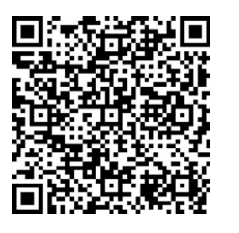



Abbreviations and Acronyms
$\mathrm{BED}=$ biologically effective dose
$\mathrm{CI}=$ confidence interval
HR = hazard ratio
NCDB = National Cancer Database
NSCLC $=$ non-small cell lung cancer
RTOG = Radiation Therapy Oncology Group
SBRT = stereotactic body radiotherapy

undergo surgery, or elect not to undergo surgery. Therefore, an alternative to lobectomy is needed for a subset of patients with early-stage lung cancer.

Stereotactic body radiotherapy (SBRT) has emerged as a safe and effective treatment for stage I lung tumors in patients who are not healthy enough to undergo surgery. In addition to preserving pulmonary parenchyma ${ }^{3}$ and having low treatment morbidity, ${ }^{4-6}$ the longer-term survival results have been encouraging. ${ }^{5}$ Several (albeit smaller) studies have achieved outcomes with SBRT that appear to be similar to surgical series (3-year survival of 79.6\%-85\%, 5 -year survival of $69.5 \%$ for SBRT vs $63 \%-85 \%$ for lobectomy). ${ }^{2,7-11}$ SBRT is associated with a local failure rate of $6.2 \%$ to $27 \%$. $^{7-9}$ As a result, there has been increasing interest in using SBRT in patients who are healthy enough for surgery but prefer to avoid it. ${ }^{4,7-9,12}$

Although desperately needed, a direct comparison between anatomic resection (lobectomy) and SBRT for patients with early-stage non-small cell lung cancer (NSCLC) who could tolerate surgery has proven to be challenging to perform. Prospective double-arm clinical trials that directly compare lobectomy with SBRT have failed to accrue patients. ${ }^{12}$

Retrospective studies using large databases have the potential to compare the effectiveness of lobectomy and SBRT across a broad range of patients and practice environments. A recent report from the National Cancer Database (NCDB) has shown surgery to be superior to SBRT for early-stage NSCLC. ${ }^{13}$ However, large databases are highly susceptible to an intrinsic bias against SBRT that could compromise comparative effectiveness studies. More specifically, a significant proportion of patients treated with SBRT were not healthy enough for surgery; this particularly poor health disproportionately threatens their survival after SBRT. ${ }^{14,15}$ Because most large database studies do not capture the severity of comorbidities (only presence or absence), it has not been possible to adjust for, match, or eliminate these particularly unhealthy patients treated with SBRT from analyses. . $^{13,16,17}$

One strategy to minimize the potential bias of patients treated with SBRT in poor health is to concentrate on healthy patients. The NCDB captures the clinical, treatment, and outcome data for approximately $70 \%$ of patients diagnosed with NSCLC in the United States. In addition to identifying comorbidity-free patients, the NCDB also allows patients who were not offered surgery because of health reasons to be excluded from analyses. Therefore, the NCDB provides a unique opportunity to retrospectively study healthy patients with cancer. The objective of this study was to compare the overall survival of healthy patients with clinical stage I NSCLC undergoing lobectomy or SBRT in the NCDB.

\section{MATERIALS AND METHODS \\ Data Source}

The NCDB is a joint project of the American Cancer Society and the American College of Surgeons, which captures incident cancer cases diagnosed or treated at Commission on Cancer-accredited facilities. ${ }^{18}$ NCDB data are abstracted by trained cancer registrars with strict educational requirements and are subject to various electronic edit checks and audits as detailed in Commission on Cancer publications. ${ }^{19}$ The NCDB states, "The data used in the study are derived from a de-identified NCDB file. The American College of Surgeons and the Commission on Cancer have not verified and are not responsible for the analytic or statistical methodology employed, or the conclusions drawn from these data by the investigator."

\section{Patient Selection}

The NCDB was queried for patients aged more than 20 years and diagnosed with invasive clinical stage I NSCLC from 2008 to 2012 who were treated by lobectomy or with SBRT (defined below) in accordance with our institutional review board-approved protocol with consent waived. Included patients did not have chemotherapy or radiation before lobectomy or SBRT. To account for the change from 6th to 7th editions of the American Joint Committee on Cancer lung cancer staging system (occurring in 2010), patients with clinical stage I were defined as having an overall stage group of I, IA, or IB, and a T stage of T1, T1a, T1b, or T2a, tumor size of $5 \mathrm{~cm}$ or less, an $\mathrm{N}$ stage of zero, and an $\mathrm{M}$ stage of zero. A full consort diagram is available in Figure E1.

\section{Data Elements}

The complete list of data elements available in the NCDB can be found online. ${ }^{20}$ The NCDB compiles a modified Charlson-Deyo Comorbidity index using International Classification of Diseases, 9th Revision, Clinical Modification secondary diagnosis codes for 15 different disease categories. ${ }^{20}$ The modified Charlson-Deyo index is reported on a scale of 0,1 , and $2+$.

\section{Selection of Healthy Patients}

To identify patients who were healthy, we used 2 different selection steps (Figure 1). First, we excluded patients with SBRT who were coded as "Surgery of the primary site was not recommended/performed because it was contraindicated due to patient risk factors (eg, comorbid conditions, advanced age)" in their "Reason for No Surgery" field. We then included only patients with a Charlson-Deyo comorbidity index of zero to eliminate patients with comorbidities. Of note, we performed an ancillary survival analysis of patients with a Charlson-Deyo score greater than 0 and found, as expected, that they had significantly worse survival than those with a Charlson-Deyo score of zero, supporting our use of this selection step (data not shown).

\section{Treatment Definitions}

A stringent definition of SBRT was chosen. Included patients with SBRT were (1) coded as receiving stereotactic radiosurgery in their treatment modality field and (2) received a biologically effective dose (BED) 


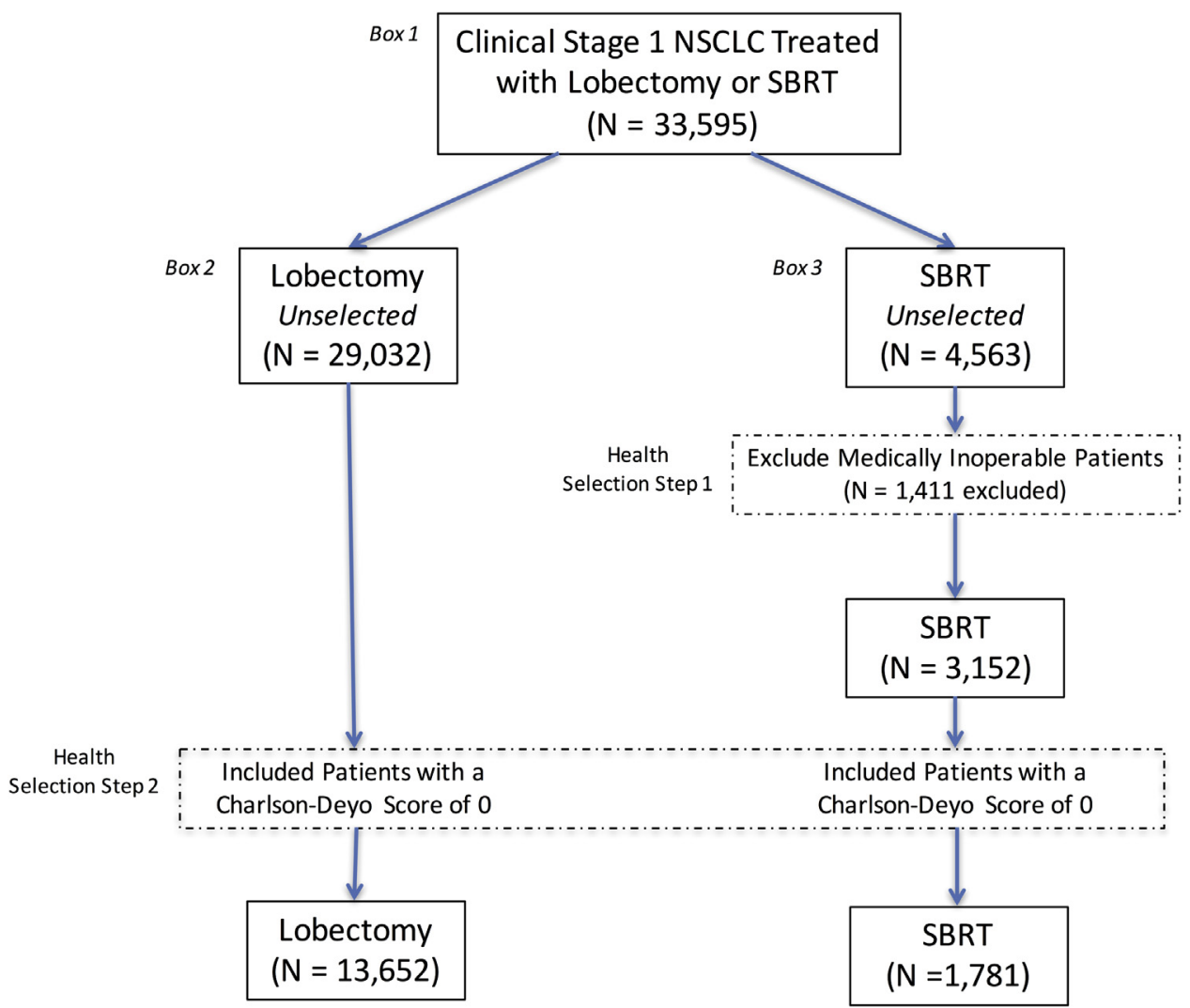

FIGURE 1. Health-based patient selection process. NSCLC, Non-small cell lung cancer; SBRT, stereotactic body radiation therapy.

of between 100 and $200 \mathrm{~Gy}$ in 3 to 5 treatment fractions to the chest/lung. ${ }^{21}$ The $\mathrm{BED}_{10}$ was calculated using the linear-quadratic model with an $\alpha / \beta$ of 10 as described in the radiation literature. ${ }^{22} \mathrm{~A}$ separate analysis was done using $\mathrm{BED}_{10}$ greater than $130 \mathrm{~Gy}$, because more recent literature has suggested this to be an even more effective dose; this restriction resulted in similar findings (Tables E1 and E2; Figure E2). ${ }^{9,21,23}$

\section{Stereotactic Body Radiotherapy in Patients Recommended to Have Surgery, but Refused}

We performed a secondary analysis comparing all patients with clinical stage I NSCLC receiving lobectomy (before any health selection steps in Figure 1) with the subset of SBRT patients who were coded as "Surgery of the primary site was not performed; it was recommended by the patient's physician, but this treatment was refused by the patient, the patient's family member, or the patient's guardian." The refusal was noted in the patient record.

\section{Statistical Analysis}

Bivariate analysis of patient characteristics was performed using chisquare tests for categoric and $t$ tests for continuous variables. Survival was measured as the number of days from the date of diagnosis to death. Survival curves were created using the Kaplan-Meier method. Survival differences were assessed using the log-rank test.

Two strategies were used to adjust for independent variables: Cox proportional hazards and propensity matching. Data elements considered included year of diagnosis, patient sex, age, race (as determined by data abstractors), Spanish/Hispanic origin, insurance payer, urban/rural status of the patient's county, median income and education level (expressed as percentage of adults with no high school diploma) of the patient's ZIP code region, tumor site, tumor size, laterality, grade, histology, clinical T stage, facility type, facility location, and treatment strategy (lobectomy, SBRT). ${ }^{20}$ Cox models were refined using backward selection with a $P$ value of .05 or less set for remaining in the model. Certain data items of clinical importance (eg, laterality, tumor site, year of diagnosis) were forced into the Cox model regardless of $P$ value. Proportional hazards assumptions were assessed using visual inspections of log-log plots of the survival function. The variable for "treatment strategy" was found to violate the proportional hazards assumption. Therefore, a time-stratified Cox model was used to evaluate the treatment effect, with the time stratification point chosen on the basis of visual inspection of unadjusted Kaplan-Meier curves (7.5 months for full cohort [Figure E3] and 15 months for the cohort of patients with SBRT who refused surgery [Figure E4]). ${ }^{16}$ The distribution of histology and grade differed between the surgery and SBRT groups. We presumed this to reflect the smaller amount of tissue for pathologists to review (eg, fine-needle aspiration) in the SBRT group. ${ }^{24,25}$ A sensitivity analysis excluding all patients with unknown grade or "other" histology demonstrated similar results (data not shown). The addition of a propensity score to these Cox models did not significantly alter any findings (Cox models available on request).

\section{Propensity Score Matching}

We created a population of propensity-matched patients with SBRT and lobectomy using the MatchIt package with nearest-neighbor 1-to-1 matching in $\mathrm{R}$ (version 3.1.1) for the full SBRT cohort and the subset of patients who were recommended to have surgery but refused. Models 
had c-statistics of 0.73 and 0.55 , respectively, and created 1781 and 256 well-matched pairs, respectively. Subjects were matched on facility type, facility location, age, sex, race, Spanish/Hispanic origin, insurance type, median income, education level, urban/rural status, Charlson score, year of diagnosis, tumor laterality, histology, tumor size, tumor location, and clinical $\mathrm{T}$ stage. Grade was excluded from the propensity matches because it was "unknown" in more than $50 \%$ of patients who received SBRT, likely reflecting a paucity of tissue from fine-needle aspiration. ${ }^{24,25}$ The standardized difference of each variable in the full cohort was assessed and found to be 0.1 or less (except for grade and histology), indicating a well-matched sample. ${ }^{26}$ Likewise, the standardized difference of each variable in the cohort of patients who received SBRT who refused surgery was found to be 0.1 or less, with the exception of grade, histology, urban/rural status, and education level (the $P$ values were insignificant for all variables except grade). Survival within the propensity-matched cohorts was compared using the Kaplan-Meier survival method and the log-rank test. All other data analysis was performed using SAS 9.4 (SAS Institute Inc, Cary, NC). All reported $P$ values are 2 tailed.

This work was funded by the James G. Hirsch, MD, endowed medical student research fellowship at Yale University School of Medicine, awarded to JER. The funding source played no role in the design of the study, the collection, analysis, or interpretation of data, the writing of the manuscript, or the decision to submit the manuscript for publication.

\section{RESULTS}

\section{Patients and Treatment}

A total of 13,652 comorbidity-free patients with clinical stage I NSCLC treated with lobectomy were compared with 1781 relatively healthy patients treated with SBRT (Figure 1). In general, the patients with SBRT tended to be older, were more likely to have Medicare, were more likely to be treated at academic facilities, and were less likely to have adenocarcinoma (Table 1). Patients with SBRT had a greater time from diagnosis to treatment than patients with lobectomy (33 vs 72 days, $P<.001$ ).

\section{Adjusted Survival Analyses}

Multivariable Cox proportional hazards models were built to adjust for potential differences in the 2 cohorts. Separate models were built for patients with clinical T1 and $\mathrm{T} 2$ tumors to better characterize the relationship within each T-staging subset (Tables 2 and 3). The relationship between SBRT and lobectomy changed over time with an important transition occurring at the 7.5-month time period (see "Materials and Methods"). This transition likely reflects differences in treatment-associated morbidity between surgery and SBRT (with surgery being more morbid). In the first 7.5 months, there were no differences in survival between surgery and SBRT for cT1 (hazard ratio $[\mathrm{HR}], 1.14 ; 95 \%$ confidence interval $[\mathrm{CI}], 0.86-1.50$; $P=.37)$ or cT2 (HR, $1.30 ; 95 \%$ CI, 0.83-2.04; $P=.26$ ) tumors. However, beyond 7.5 months, lobectomy offered significantly improved survival compared with SBRT for cT1 (HR, 0.38; 95\% CI, 0.33-0.43; $P<.001$ ) and cT2 (HR, $0.38 ; 95 \%$ CI, $0.31-0.46 ; P<.001$ ) tumors.
As an alternate adjustment strategy, propensity matching was performed within the healthier lobectomy and SBRT cohorts, resulting in 1781 well-balanced pairs (Table 1). The median follow-up for surviving patients was 28.6 months (interquartile range, 20.7) for SBRT cases and 31.6 months (interquartile range, 23.1) for lobectomy cases. Kaplan-Meier survival analysis of propensitymatched patients revealed lobectomy to be associated with a superior 5 -year survival $(59 \%$ vs $29 \%, P<.001)$ and median survival (71 months; $95 \%$ CI, 86-unmet months vs survival 39 months; $95 \%$ CI, 37-41 months) compared with SBRT (Figure 2, $A$ ).

\section{Survival of Patients With Stereotactic Body Radiotherapy Recommended to Have Surgery, but Refused}

A small subset $(n=235)$ of the unselected patients with SBRT (ie, before selection steps for healthy patients; Figure 1, box 3) were categorized in the NCDB as having been recommended to have surgery, but had refused (see "Materials and Methods"). These patients were propensity matched with 29,032 unselected patients with lobectomy (Figure 1, box 2), resulting in 235 well-matched pairs (Table E3). Lobectomy was associated with superior 5year survival (58\% vs $40 \%$ for SBRT, $P=.010)$ and median survival (71 vs 53 months for SBRT) (Figure 2, B). In a multivariable Cox analysis, there was no significant difference between lobectomy and SBRT in the first 15 months (HR, 1.06; 95\% CI, 0.71-1.57; $P=.78$ ); however, beyond 15 months, lobectomy offered significantly improved survival compared with SBRT (HR, 0.59; 95\% CI, 0.450.79; $P<.001$ ) (Table E4).

\section{Upstaging and Additional Treatment}

One of the proposed advantages of lobectomy over SBRT has been the ability to identify radiographically occult lymph node metastases, allowing the node-positive patients to benefit from adjuvant chemotherapy. ${ }^{27-29}$ Lymph node metastases were discovered in the surgical specimens of $12 \%$ (1633) of the patients who underwent lobectomy (all clinically node-negative), of whom $70 \%$ received postoperative chemotherapy.

Two additional indications for adjuvant chemotherapy after lobectomy are tumors greater than $4 \mathrm{~cm}$ and positive margins. ${ }^{30,31}$ Chemotherapy was given to 398 patients who underwent lobectomy with tumors greater than $4 \mathrm{~cm}$ and 53 patients with positive margins, whereas 538 patients received adjuvant chemotherapy for an unknown indication. Overall, $16 \%$ (2126) of patients with lobectomy received adjuvant chemotherapy.

By comparison, only $2 \%$ of patients (31) with SBRT received chemotherapy after SBRT. Among patients with tumors larger than $4 \mathrm{~cm}, 8$ of $142(6 \%)$ received chemotherapy after SBRT. 
TABLE 1. Bivariate analysis of unmatched and propensity-matched stereotactic body radiation therapy and lobectomy cohorts

\begin{tabular}{|c|c|c|c|c|c|c|}
\hline & \multicolumn{3}{|c|}{ Unmatched cohort } & \multicolumn{3}{|c|}{ Matched cohort } \\
\hline & $\begin{array}{c}\begin{array}{c}\text { Lobectomy } \\
(N=13,652)\end{array} \\
\end{array}$ & $\begin{array}{c}\text { SBRT } \\
(\mathbf{N}=1781) \\
\end{array}$ & & $\begin{array}{l}\text { Lobectomy } \\
(\mathrm{N}=1781) \\
\end{array}$ & $\begin{array}{c}\text { SBRT } \\
(\mathbf{N}=\mathbf{1 7 8 1}) \\
\end{array}$ & \\
\hline & $\mathbf{N}(\%)$ & $\mathbf{N}(\%)$ & $P$ value & $\mathbf{N}(\%)$ & $\mathbf{N}(\%)$ & $P$ value \\
\hline Year of diagnosis & & & $<.001$ & & & .99 \\
\hline 2008 & $2372(17)$ & $177(10)$ & & $176(10)$ & $177(10)$ & \\
\hline 2009 & $2741(20)$ & $283(16)$ & & $278(16)$ & $283(16)$ & \\
\hline 2010 & $2732(20)$ & 347 (19) & & $358(20)$ & 347 (19) & \\
\hline 2011 & $2840(21)$ & $436(24)$ & & $427(24)$ & $436(24)$ & \\
\hline 2012 & $2967(22)$ & $538(30)$ & & $542(30)$ & $538(30)$ & \\
\hline Sex & & & .17 & & & .73 \\
\hline $\mathrm{F}$ & $7541(55)$ & $1014(57)$ & & $1004(56)$ & $1014(57)$ & \\
\hline M & $6111(45)$ & $767(43)$ & & 777 (44) & $767(43)$ & \\
\hline \multicolumn{7}{|l|}{ Age (y) } \\
\hline Mean (SD) & $66.6(10.2)$ & $75.5(9.1)$ & $<.001$ & $74.8(7.8)$ & $75.5(9.1)$ & .03 \\
\hline Race & & & $<.001$ & & & .73 \\
\hline Nonwhite & 1714 (13) & $165(9)$ & & $171(10)$ & $165(9)$ & \\
\hline White & $11,938(87)$ & $1616(91)$ & & $1610(90)$ & $1616(91)$ & \\
\hline Spanish Hispanic origin & & & .003 & & & .85 \\
\hline Not Spanish/Hispanic & $12,512(92)$ & $1647(92)$ & & $1638(92)$ & $1647(92)$ & \\
\hline Spanish/Hispanic origin & $361(3)$ & $24(1)$ & & $25(1)$ & $24(1)$ & \\
\hline Unknown & $779(6)$ & $110(6)$ & & $118(7)$ & $110(6)$ & \\
\hline Primary payer & & & $<.001$ & & & .084 \\
\hline Medicaid & $588(4)$ & $52(3)$ & & $58(3)$ & $52(3)$ & \\
\hline Medicare & $7642(56)$ & $1400(79)$ & & $1390(78)$ & $1400(79)$ & \\
\hline No insurance & $320(2)$ & $16(1)$ & & $23(1)$ & $16(1)$ & \\
\hline Other government & $142(1)$ & $61(3)$ & & $35(2)$ & $61(3)$ & \\
\hline Private insurance/managed care & $4768(35)$ & $228(13)$ & & $250(14)$ & $228(13)$ & \\
\hline Unknown & $192(1)$ & $24(1)$ & & $25(1)$ & $24(1)$ & \\
\hline Median income quartiles & & & .002 & & & .85 \\
\hline$\$ 48,000+$ & $7912(58)$ & $955(54)$ & & $970(54)$ & $955(54)$ & \\
\hline$<\$ 48,000$ & $5584(41)$ & $806(45)$ & & $793(45)$ & $806(45)$ & \\
\hline Unknown & $156(1)$ & $20(1)$ & & $18(1)$ & $20(1)$ & \\
\hline Percent no high school degree & & & .12 & & & .67 \\
\hline$\geq 21 \%$ & $2070(15)$ & $236(13)$ & & $207(12)$ & $236(13)$ & \\
\hline $13 \%-20.9 \%$ & $3612(26)$ & $513(29)$ & & $523(29)$ & $513(29)$ & \\
\hline $7 \%-12.9 \%$ & $4661(34)$ & $611(34)$ & & $627(35)$ & $611(34)$ & \\
\hline$<7 \%$ & $3154(23)$ & $401(23)$ & & $406(23)$ & $401(23)$ & \\
\hline Unknown & $155(1)$ & $20(1)$ & & $18(1)$ & $20(1)$ & \\
\hline Urban/rural & & & .56 & & & .99 \\
\hline Metro & $10,962(80)$ & $1422(80)$ & & $1415(79)$ & $1422(80)$ & \\
\hline Rural & $285(2)$ & $38(2)$ & & $39(2)$ & $38(2)$ & \\
\hline Unknown & $441(3)$ & $49(3)$ & & $52(3)$ & $49(3)$ & \\
\hline Urban & $1964(14)$ & $272(15)$ & & $275(15)$ & $272(15)$ & \\
\hline Facility type & & & $<.001$ & & & .44 \\
\hline Academic/research program (including NCI) & $4479(33)$ & $846(48)$ & & $823(46)$ & $846(48)$ & \\
\hline Nonacademic program & $9173(67)$ & $935(52)$ & & $958(54)$ & $935(52)$ & \\
\hline Facility location & & & $<.001$ & & & .84 \\
\hline Central & $5781(42)$ & 783 (44) & & $811(46)$ & $783(44)$ & \\
\hline Mountain & $556(4)$ & $85(5)$ & & $78(4)$ & $85(5)$ & \\
\hline North East & $2348(17)$ & 324 (18) & & 306 (17) & 324 (18) & \\
\hline Pacific & 1457 (11) & $119(7)$ & & $114(6)$ & $119(7)$ & \\
\hline South Atlantic & $3510(26)$ & $470(26)$ & & $472(27)$ & $470(26)$ & \\
\hline Primary site & & & $<.001$ & & & .82 \\
\hline Lower lobe & $4358(32)$ & $515(29)$ & & $509(29)$ & $515(29)$ & \\
\hline
\end{tabular}




\begin{tabular}{|c|c|c|c|c|c|c|}
\hline & \multicolumn{3}{|c|}{ Unmatched cohort } & \multicolumn{3}{|c|}{ Matched cohort } \\
\hline & $\begin{array}{c}\text { Lobectomy } \\
(\mathrm{N}=\mathbf{1 3 , 6 5 2})\end{array}$ & $\begin{array}{c}\text { SBRT } \\
(\mathbf{N}=\mathbf{1 7 8 1})\end{array}$ & & $\begin{array}{l}\text { Lobectomy } \\
(N=1781)\end{array}$ & $\begin{array}{c}\text { SBRT } \\
(\mathbf{N}=\mathbf{1 7 8 1})\end{array}$ & \\
\hline & $\mathbf{N}(\%)$ & $\mathbf{N}(\%)$ & $P$ value & $\mathbf{N}(\%)$ & $\mathbf{N}(\%)$ & $P$ value \\
\hline Lung, NOS & $184(1)$ & $53(3)$ & & $47(3)$ & $53(3)$ & \\
\hline Main bronchus & $21(0)$ & $\sim$ & & $\sim$ & $\sim$ & \\
\hline Middle lobe & $730(5)$ & $92(5)$ & & $97(5)$ & $92(5)$ & \\
\hline Overlapping lesion & $88(1)$ & $\sim$ & & $\sim$ & $\sim$ & \\
\hline Upper lobe & $8271(61)$ & $1118(63)$ & & $1124(63)$ & $1118(63)$ & \\
\hline Laterality & & & .003 & & & .79 \\
\hline Left & $5507(40)$ & $792(44)$ & & $776(44)$ & $792(44)$ & \\
\hline Other* & $29(0)$ & $\sim$ & & $\sim$ & $\sim$ & \\
\hline Right & $8116(59)$ & $987(55)$ & & $1002(56)$ & $987(55)$ & \\
\hline Histology & & & $<.001$ & & & $<.001$ \\
\hline Adenocarcinoma & $9379(69)$ & $850(48)$ & & $886(50)$ & $850(48)$ & \\
\hline Large cell carcinoma & $317(2)$ & $17(1)$ & & $21(1)$ & $17(1)$ & \\
\hline Other NSCLC & $454(3)$ & 331 (19) & & $228(13)$ & $331(19)$ & \\
\hline Squamous cell carcinoma & $3502(26)$ & $583(33)$ & & $646(36)$ & $583(33)$ & \\
\hline Grade $\dagger$ & & & $<.001$ & & & $<.001$ \\
\hline 1 & 1937 (14) & $129(7)$ & & $223(13)$ & $129(7)$ & \\
\hline 2 & 6635 (49) & $280(16)$ & & $846(48)$ & $280(16)$ & \\
\hline 3 & $4361(32)$ & $378(21)$ & & $598(34)$ & $378(21)$ & \\
\hline 4 & $174(1)$ & $\sim$ & & $20(1)$ & $\sim$ & \\
\hline Unknown & $545(4)$ & $985(55)$ & & $94(5)$ & 985 (55) & \\
\hline Tumor size (mm) & & & $<.001$ & & & .82 \\
\hline Mean (SD) & $25.4(10.5)$ & $23.8(9.3)$ & & $23.7(10.0)$ & $23.8(9.3)$ & \\
\hline Clinical $\mathrm{T}$ stage & & & $<.001$ & & & .90 \\
\hline 1 & $9543(70)$ & $1371(77)$ & & $1374(77)$ & $1371(77)$ & \\
\hline 2 & 4109 (30) & $410(23)$ & & 407 (23) & $410(23)$ & \\
\hline \multicolumn{7}{|l|}{ 30-d mortality } \\
\hline No & $13,153(96)$ & & & $1679(94)$ & & \\
\hline Yes & $252(2)$ & & & $58(3)$ & & \\
\hline Unknown & $247(2)$ & & & $44(2)$ & & \\
\hline \multicolumn{7}{|l|}{ 90-d mortality } \\
\hline No & $12,856(94)$ & & & $1632(92)$ & & \\
\hline Yes & $449(3)$ & & & $92(5)$ & & \\
\hline Unknown & 347 (3) & & & $57(3)$ & & \\
\hline
\end{tabular}

\section{DISCUSSION}

Attempts to compare the efficacy of SBRT and lobectomy for early-stage lung cancer have proven challenging to complete and have yielded mixed results. ${ }^{12,13,16,17,32}$ Two prospective trials of SBRT in surgery-eligible patients (single arm) have closed (Japan Clinical Oncology Group 0403 and Radiation Therapy Oncology Group [RTOG] 0618). Preliminary results suggested a concerning degree of local failure (2-year local failure rate in the RTOG 0618 trial was $19 \%$ ) but acceptable short-term overall survival (2-year survival in RTOG 0618 84.4\%). ${ }^{7,33}$ Prospective double arm trials comparing surgery with SBRT in healthier patients (STARS, ROSEL, and American
College of Surgeons Oncology Group Z4099 trials) have failed to accrue patients. A recent pooled analysis of the results from STARS and ROSEL (27 surgery and 31 SBRT cases) indicated superior survival in the SBRT cohort at 3 years. ${ }^{12}$

More recently, 2 large database studies using the Surveillance Epidemiology and End Results Medicare and the NCDB have shown a significant survival advantage to surgery over SBRT. ${ }^{13,17}$ On the other hand, the advantage of surgery in an earlier Surveillance Epidemiology and End Results Medicare study was less clear. ${ }^{16}$

Unfortunately, retrospective studies are particularly problematic for the SBRT/lobectomy comparison because 
TABLE 2. Cox proportional hazards model on 10,914 healthy patients with clinical stage T1 non-small cell lung cancer

\begin{tabular}{|c|c|c|c|c|}
\hline Covariate & Level & $\mathbf{N}$ & HR (LCL, UCL) & $P$ value \\
\hline \multirow[t]{2}{*}{ Treatment effect up to $7.5 \mathrm{mo}$ from diagnosis } & SBRT & 1371 & Reference & \\
\hline & Lobectomy & 9543 & $1.14(0.86,1.50)$ & .37 \\
\hline \multirow[t]{2}{*}{ Treatment effect after $7.5 \mathrm{mo}$ from diagnosis } & SBRT & 1371 & Reference & \\
\hline & Lobectomy & 9543 & $0.38(0.33,0.43)$ & $<.001$ \\
\hline \multirow[t]{5}{*}{ Year of diagnosis } & 2008 & 1729 & Reference & \\
\hline & 2009 & 2042 & $1.02(0.91,1.14)$ & .75 \\
\hline & 2010 & 2211 & $0.82(0.73,0.93)$ & .002 \\
\hline & 2011 & 2382 & $0.90(0.79,1.03)$ & .13 \\
\hline & 2012 & 2550 & $0.88(0.76,1.02)$ & .081 \\
\hline \multirow[t]{2}{*}{ Facility type } & Academic & 3810 & Reference & \\
\hline & Nonacademic & 7104 & $1.20(1.10,1.30)$ & .002 \\
\hline \multirow[t]{2}{*}{ Sex } & $\mathrm{F}$ & 6254 & Reference & \\
\hline & M & 4660 & $1.45(1.34,1.57)$ & $<.001$ \\
\hline Age & & & $1.02(1.02,1.03)$ & $<.001$ \\
\hline \multirow[t]{6}{*}{ Primary payer } & Private insurance & 3573 & Reference & \\
\hline & Medicaid & 435 & $1.59(2.37,2.24)$ & $<.001$ \\
\hline & Medicare & 6376 & $1.19(1.49,1.54)$ & $<.001$ \\
\hline & No insurance & 227 & $1.03(1.95,2.44)$ & .034 \\
\hline & Other government & 148 & $0.87(1.73,2.10)$ & .25 \\
\hline & Unknown & 155 & $1.20(2.31,2.03)$ & .002 \\
\hline \multirow[t]{6}{*}{ Primary site } & Lower lobe & 3337 & Reference & \\
\hline & Lung, NOS & 169 & $1.03(0.78,1.37)$ & .83 \\
\hline & Main bronchus & 17 & $1.05(0.09,11.65)$ & .97 \\
\hline & Middle lobe & 607 & $1.03(0.86,1.23)$ & .75 \\
\hline & Overlapping lesion & 44 & $2.24(1.38,3.63)$ & .001 \\
\hline & Upper lobe & 6740 & $0.98(0.90,1.07)$ & .67 \\
\hline \multirow[t]{3}{*}{ Laterality } & Left & 4462 & Reference & \\
\hline & Other* & 22 & $0.68(0.09,4.82)$ & .70 \\
\hline & Right & 6430 & $1.07(0.98,1.16)$ & .12 \\
\hline \multirow[t]{4}{*}{ Histology } & Adenocarcinoma & 7478 & Reference & \\
\hline & Large cell carcinoma & 225 & $1.39(1.06,1.82)$ & .015 \\
\hline & Other NSCLC & 546 & $1.37(1.18,1.60)$ & $<.001$ \\
\hline & Squamous cell carcinoma & 2665 & $1.17(1.07,1.28)$ & $<.001$ \\
\hline \multirow[t]{5}{*}{ Grade } & 1 & 1631 & Reference & \\
\hline & 2 & 4971 & $1.61(1.38,1.87)$ & $<.001$ \\
\hline & 3 & 3061 & $1.75(1.50,2.05)$ & $<.001$ \\
\hline & 4 & 112 & $1.55(1.02,2.34)$ & .038 \\
\hline & Unknown & 1139 & $1.75(1.45,2.11)$ & $<.001$ \\
\hline Tumor size & & & $1.02(1.02,1.03)$ & $<.001$ \\
\hline
\end{tabular}

Note that some n values are censored with a " " in accordance with NCDB policy on reporting cells with low n values. $H R$, Hazard ratio; $L C L$, lower confidence limit; $U C L$, upper confidence limit; $S B R T$, stereotactic body radiation therapy; $F$, female; $M$, male; NOS, not otherwise specified; NSCLC, non-small cell lung cancer. *Other laterality designates main bronchus and other central tumors.

of a health-related bias against SBRT. Poor health is the most common reason that patients are ineligible for surgery, and these same health concerns can independently compromise long-term survival. ${ }^{14,15}$ Over the past decade, medically inoperable patients have been increasingly referred for SBRT therapy, and as a result, the SBRT population in most cancer registries likely has a significant proportion of particularly unhealthy (medically inoperable) patients. ${ }^{32}$ In fact, before our health-based selection, $30 \%$ of patients with clinical stage I treated with SBRT in the NCDB were documented to be medically inoperable (Figure 1, selection step \#1). The exclusion of patients with particularly poor health is a critical difference between the current study and the recent NCDB study by Puri and colleagues, ${ }^{13}$ which included these patients in their analyses; 3-year survival for SBRT in the study by Puri and colleagues was $46 \%{ }^{13}$ but is $53 \%$ in the current study.

Large databases do not capture the severity of medical conditions (ie, administrative/registry databases only capture whether or not a condition is present), making it difficult to adjust for the particularly poor health that is over-represented in the SBRT cohort. Propensity matching, although technically feasible, is intrinsically flawed because the medically inoperable patient who received 
TABLE 3. Cox proportional hazards model on 4519 healthy patients with clinical stage $T 2$ non-small cell lung cancer

\begin{tabular}{|c|c|c|c|c|}
\hline Covariate & Level & $\mathbf{N}$ & HR (LCL, UCL) & $P$ value \\
\hline \multirow{2}{*}{$\begin{array}{l}\text { Treatment effect up to } 7.5 \mathrm{mo} \\
\text { from diagnosis }\end{array}$} & SBRT & 410 & Reference & \\
\hline & Lobectomy & 4109 & $1.30(0.83,2.04)$ & .26 \\
\hline \multirow{2}{*}{$\begin{array}{l}\text { Treatment effect after } 7.5 \mathrm{mo} \\
\text { from diagnosis }\end{array}$} & SBRT & 410 & Reference & \\
\hline & Lobectomy & 4109 & $0.38(0.31,0.46)$ & $<.001$ \\
\hline \multirow[t]{5}{*}{ Year of diagnosis } & 2008 & 820 & Reference & \\
\hline & 2009 & 982 & $0.99(0.85,1.14)$ & .84 \\
\hline & 2010 & 868 & $0.92(0.79,1.08)$ & .33 \\
\hline & 2011 & 894 & $0.92(0.78,1.10)$ & .36 \\
\hline & 2012 & 955 & $0.84(0.69,1.03)$ & .10 \\
\hline \multirow[t]{2}{*}{ Facility type } & Academic & 1515 & Reference & \\
\hline & Nonacademic & 3004 & $1.28(1.14,1.44)$ & $<.001$ \\
\hline \multirow[t]{2}{*}{ Sex } & $\mathrm{F}$ & 2301 & Reference & \\
\hline & M & 2218 & $1.40(1.25,1.55)$ & $<.001$ \\
\hline Age & & & $1.02(1.02,1.03)$ & $<.001$ \\
\hline \multirow[t]{3}{*}{ Median income } & $<\$ 48,000$ & 1936 & Reference & \\
\hline & $\$ 48,000+$ & 2523 & $0.99(0.88,1.10)$ & .82 \\
\hline & Unknown & 60 & $2.15(1.40,3.30)$ & $<.001$ \\
\hline \multirow[t]{6}{*}{ Primary payer } & Private insurance & 1423 & Reference & \\
\hline & Medicaid & 205 & $1.34(1.02,1.75)$ & .033 \\
\hline & Medicare & 2666 & $1.19(1.04,1.39)$ & .015 \\
\hline & No insurance & 109 & $0.70(0.43,1.13)$ & .14 \\
\hline & Other Government & 55 & $0.92(0.56,1.52)$ & .75 \\
\hline & Unknown & 61 & $1.00(0.60,1.65)$ & .99 \\
\hline \multirow[t]{4}{*}{ Urban/rural } & Rural & 77 & Reference & \\
\hline & Metro & 3601 & $1.24(0.81,1.89)$ & .33 \\
\hline & Unknown & 134 & $2.24(1.34,3.74)$ & .002 \\
\hline & Urban & 707 & $1.38(0.89,2.14)$ & .15 \\
\hline \multirow[t]{6}{*}{ Primary site } & Lower lobe & 1536 & Reference & \\
\hline & Lung, NOS & 68 & $1.20(0.82,1.75)$ & .36 \\
\hline & Main bronchus & $\sim$ & $0.23(0.02,2.59)$ & .24 \\
\hline & Middle lobe & 215 & $1.08(0.84,1.38)$ & .57 \\
\hline & Overlapping lesion & 46 & $1.18(0.73,1.89)$ & .50 \\
\hline & Upper lobe & 2649 & $0.91(0.81,1.02)$ & .099 \\
\hline \multirow[t]{3}{*}{ Laterality } & Left & 1837 & Reference & \\
\hline & Other* & $\sim$ & $2.38(0.59,9.62)$ & .22 \\
\hline & Right & 2673 & $0.95(0.85,1.05)$ & .32 \\
\hline \multirow[t]{4}{*}{ Histology } & Adenocarcinoma & 2751 & Reference & \\
\hline & Large cell carcinoma & 109 & $1.91(1.38,2.64)$ & $<.001$ \\
\hline & Other NSCLC & 239 & $1.17(0.94,1.4)$ & .15 \\
\hline & Squamous cell carcinoma & 1420 & $1.19(1.06,1.33)$ & .004 \\
\hline \multirow[t]{5}{*}{ Grade } & 1 & 435 & Reference & \\
\hline & 2 & 1944 & $1.64(1.30,2.06)$ & $<.001$ \\
\hline & 3 & 1678 & $1.78(1.41,2.25)$ & $<.001$ \\
\hline & 4 & 71 & $0.90(0.52,1.53)$ & .69 \\
\hline & Unknown & 391 & $1.45(1.09,1.93)$ & .011 \\
\hline Tumor size & & & $1.01(1.01,1.02)$ & $<.001$ \\
\hline
\end{tabular}

Note that some n-values are censored with a " $\sim$ " in accordance with NCDB policy on reporting cells with low n-values. $H R$, Hazard ratio; $L C L$, lower confidence limit; $U C L$, upper confidence limit; $S B R T$, stereotactic body radiation therapy; $F$, female; $M$, male; NOS, not otherwise specified; NSCLC, non-small cell lung cancer. * Other laterality designates main bronchus and other central tumors.

SBRT has no true counterpart in the surgery cohort (even if a completely "balanced" population can be created among matching variables). The current study is the first to take an approach of trying to eliminate these patients in particularly poor health who received SBRT by using a more common data field (comorbidity index of " 0 ") and a data field that specifically indicates the "reason for no surgery." As a result, we believe this study represents the least-biased retrospective comparison of SBRT to lobectomy for clinical stage I NSCLC in a large database, and supports the use of lobectomy in patients without a medical condition that precludes surgery. 

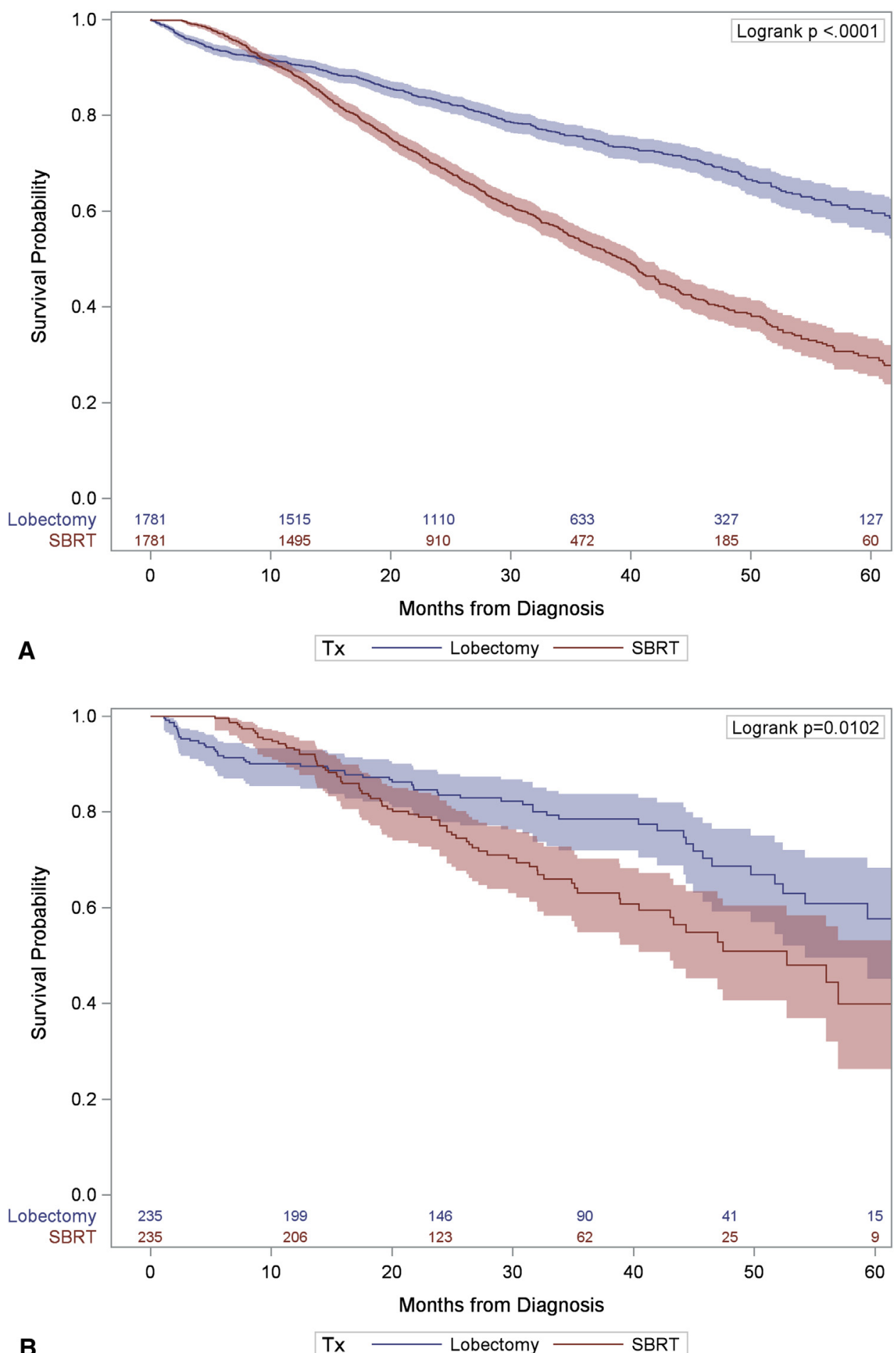

FIGURE 2. Survival curves (from diagnosis) of healthy patients with clinical stage I NSCLC derived by the Kaplan-Meier method. Propensity-matched "healthy" patients with lobectomy and SBRT (A) and propensity-matched patients with lobectomy and patients with SBRT (B) who were recommended to have surgery but refused. SBRT, Stereotactic body radiation therapy; $T x$, treatment.

Of note, the small subset of patients who received SBRT and were recommended to have surgery, but refused, fared better than our "healthy" SBRT cohort (which had eliminated medically inoperable patients and those with any comorbidity). Although this group of patients is small, this result suggests that the larger "healthy" cohort (Figure 1, box) cohort may still contain some unhealthy patients who were treated with SBRT. This is a critical 
point, because the difference in 5-year survival between the 2 analyses (29\% for "healthy" patients with SBRT and $40 \%$ for patients with SBRT who refused a recommended surgery) may be enough to alter patient decision making, even if surgery were to remain statistically superior.

We attempted to understand the nature of the survival differences between the SBRT and lobectomy groups by evaluating the use of adjuvant therapy. The use of adjuvant chemotherapy was more common in the surgery cohort $(16 \%$ vs $2 \%$ of patients with SBRT). Chemotherapy is known to offer an approximately 5\% increase in 5-year survival for the indications listed. ${ }^{28,29}$ However, if updated data from the LACE meta-analysis ( $11 \%$ increase in 5-year survival for stage II NSCLC) was applied to our dataset, it can be estimated that adjuvant chemotherapy would have prevented 132 patient deaths in the unmatched lobectomy group by the 5-year mark. However, adding 132 deaths to the lobectomy cohort (albeit an extremely simplistic test for impact) would reduce the 5-year overall survival by only $1 \%{ }^{34}$ Therefore, the discrepancy in the use of chemotherapy (potentially driven by the surgical lymph node evaluation) likely made a modest contribution to the overall survival difference between the lobectomy and SBRT groups.

\section{Study Limitations}

The current study has several limitations in addition to those traditionally associated with retrospective database analyses. In addition to selection bias that is not fully characterized, it is unclear precisely how healthy the "healthy" cohort really was. The study design was focused on excluding patients who were not healthy enough for surgery; however, the residual population may have not been universally considered healthy, despite a lack of documented comorbidities. For example, the SBRT group had a significantly higher mean age than the lobectomy group, possibly indicating health differences that were not captured in our selections. Furthermore, it is unclear how the "reason for no surgery" field in the NCDB was informed (eg, surgeon, primary care). Therefore, some medically inoperable patients may have remained in the SBRT cohort, and some healthy patients with SBRT may have been excluded. In addition, the NCDB does not capture cause of death or information on cancer recurrence. Therefore, overall survival was examined, but not cancerspecific survival or recurrence-free survival. As a result, this study is unable to determine the extent to which observed survival differences reflect more effective cancer treatment.

The accuracy of the clinical staging evaluation also could contribute to observed survival differences, because a disproportionate inclusion of understaged patients (poorer prognosis) could skew results. Lymph nodes were evaluated by a pathologist in only $6 \%$ of the patients who received
SBRT (presumably during pretreatment staging). The NCDB does not characterize the nature of the lymph node evaluation before lobectomy (ie, mediastinoscopy). Therefore, it is unknown whether surgery cases underwent a more thorough nodal staging workup before treatment. However, it is worth noting that invasive nodal staging would not have been recommended before lobectomy for the majority of the patients in the surgery group per American College of Chest Physicians Evidence-Based Clinical Practice Guidelines. $^{35}$

\section{CONCLUSIONS}

The current study findings suggest that lobectomy leads to superior long-term survival over SBRT in patients with clinical stage I NSCLC whose health does not prohibit the use of surgery; however, the magnitude of the survival difference is unclear, because separate strategies to eliminate the bias of poor health in the SBRT cohort have generated very different SBRT survival estimates. Nevertheless, because all estimates of SBRT survival were significantly lower than that for lobectomy, these data indicate that surgery may be the preferred modality in patients with clinical stage I NSCLC who are healthy enough to undergo it. This study further highlights the urgent need for a prospective clinical trial with sufficient patient accrual to answer this important question.

\section{Conflict of Interest Statement}

R.H.D. reports grants from 21st Century Oncology and Merck \& Co, outside of the submitted work, and is a consultant for AMAG Pharmaceuticals and Leidos Biomedical. J.B.Y. reports grants from 21st Century Oncology, outside of the submitted work. All other authors have nothing to disclose with regard to commercial support.

This publication was made possible by the James G. Hirsch, MD, endowed medical student research fellowship at Yale University School of Medicine, awarded to JER. The funding source played no role in the design of the study, the collection, analysis, or interpretation of data, the writing of the manuscript, or the decision to submit the manuscript for publication.

\section{References}

1. American Cancer Society. Cancer Facts and Figures 2015. Atlanta, GA: American Cancer Society; 2015.

2. Ginsberg RJ, Rubinstein LV. Randomized trial of lobectomy versus limited resection for T1 N0 non-small cell lung cancer. Lung Cancer Study Group. Ann Thorac Surg. 1995;60:615-23.

3. Stanic S, Paulus R, Timmerman RD, Michalski JM, Barriger RB, Bezjak A, et al No clinically significant changes in pulmonary function following stereotactic body radiation therapy for early- stage peripheral non-small cell lung cancer: an analysis of RTOG 0236. Int J Radiat Oncol Biol Phys. 2014;88:1092-9.

4. Crabtree TD, Denlinger CE, Meyers BF, Naqa El I, Zoole J, Krupnick AS, et al. Stereotactic body radiation therapy versus surgical resection for stage I nonsmall cell lung cancer. J Thorac Cardiovasc Surg. 2010;140:377-86.

5. Timmerman R, Paulus R, Galvin J, Michalski J, Straube W, Bradley J, et al. Stereotactic body radiation therapy for inoperable early stage lung cancer JAMA. 2010;303:1070-6. 
6. Grills IS, Mangona VS, Welsh R, Chmielewski G, McInerney E, Martin S, et al Outcomes after stereotactic lung radiotherapy or wedge resection for stage I nonsmall-cell lung cancer. J Clin Oncol. 2010;28:928-35.

7. Lagerwaard FJ, Verstegen NE, Haasbeek CJ, Slotman BJ, Paul MA, Smit EF, et al. Outcomes of stereotactic ablative radiotherapy in patients with potentially operable stage I non-small cell lung cancer. Int J Radiat Oncol Biol Phys. 2012; 83:348-53.

8. Verstegen NE, Oosterhuis JW, Palma DA, Rodrigues G, Lagerwaard FJ, van der Elst A, et al. Stage I-II non-small-cell lung cancer treated using either stereotactic ablative radiotherapy (SABR) or lobectomy by video-assisted thoracoscopic surgery (VATS): outcomes of a propensity score-matched analysis. Ann Oncol. 2013;24:1543-8.

9. Onishi H, Shirato H, Nagata Y, Hiraoka M, Fujino M, Gomi K, et al. Stereotactic body radiotherapy (SBRT) for operable stage I non-small-cell lung cancer: can SBRT be comparable to surgery? Int J Radiat Oncol Biol Phys. 2011;81:1352-8.

10. van Rens M, la Riviere de AB. Prognostic assessment of 2,361 patients who underwent pulmonary resection for non-small cell lung cancer, stage I, II, and IIIA. Chest. 2000;117:374-9.

11. Yamamoto K, Ohsumi A, Kojima F, Imanishi N, Matsuoka K, Ueda M, et al. Long-term survival after video-assisted thoracic surgery lobectomy for primary lung cancer. Ann Thorac Surg. 2010;89:353-9.

12. Chang JY, Senan S, Paul MA, Mehran RJ, Louie AV, Balter P, et al. Stereotactic ablative radiotherapy versus lobectomy for operable stage I non-small cell lung cancer: a pooled analysis of two randomised trials. Lancet Oncol. 2015; 16:630-7.

13. Puri V, Crabtree TD, Bell JM, Broderick SR, Morgensztern D, Colditz GA, et al. Treatment outcomes in stage I lung cancer: a comparison of surgery and stereotactic body radiation therapy. J Thorac Oncol. 2015;10:1776-84.

14. Howlader N, Mariotto AB, Woloshin S, Schwartz LM. Providing clinicians and patients with actual prognosis: cancer in the context of competing causes of death. J Natl Cancer Inst Monographs. 2014;2014:255-64.

15. Mokhles S, Nuyttens JJ, Maat AP, Birim O, Aerts JGJV, Bogers AJJC, et al. Survival and treatment of non-small cell lung cancer stage I-II treated surgically or with stereotactic body radiotherapy: patient and tumor-specific factors affect the prognosis. Ann Surg Oncol. 2015;22:316-23.

16. Shirvani SM, Jiang J, Chang JY, Welsh JW, Gomez DR, Swisher S, et al. Comparative effectiveness of 5 treatment strategies for early-stage non-small cell lung cancer in the elderly. Int J Radiat Oncol Biol Phys. 2012;84:1060-70.

17. Yu JB, Soulos PR, Cramer LD, Decker RH, Kim AW, Gross CP. Comparative effectiveness of surgery and radiosurgery for stage I non-small cell lung cancer. Cancer. 2015;121:2341-9.

18. Rosen JE, Hancock JG, Kim AW, Detterbeck FC, Boffa DJ. Predictors of mortality after surgical management of lung cancer in the National Cancer Database. Ann Thorac Surg. 2014;98:1953-60.

19. American College of Surgeons Commission on Cancer. Cancer program standards 2012: ensuring patient-centered care. Chicago, IL: American College of Surgeons; 2011. Available at: https://www.facs.org/ /media/files/quality\% 20programs/cancer/coc/programstandards2012.ashx.

20. American College of Surgeons. The National Cancer Database 2013 PUF Data Dictionary. 2011. Available at: http://ncdbpuf.facs.org/node/259. Accessed July 1, 2015.
21. Rowe BP, Boffa DJ, Wilson LD, Kim AW, Detterbeck FC, Decker RH. Stereotactic body radiotherapy for central lung tumors. J Thorac Oncol. 2012;7: 1394-9

22. Corso CD, Park HS, Kim AW, Yu JB, Husain Z, Decker RH. Racial disparities in the use of SBRT for treating early-stage lung cancer. Lung Cancer. 2015;89: $133-8$.

23. Grills IS, Hope AJ, Guckenberger M, Kestin LL, Werner-Wasik M, Yan D, et al A collaborative analysis of stereotactic lung radiotherapy outcomes for earlystage non-small-cell lung cancer using daily online cone-beam computed tomography image-guided radiotherapy. J Thorac Oncol. 2012;7:1382-93.

24. Edwards SL, Roberts C, McKean ME, Cockburn JS, Jeffrey RR, Kerr KM. Preoperative histological classification of primary lung cancer: accuracy of diagnosis and use of the non-small cell category. J Clin Pathol. 2000;53:537-40.

25. Cataluna J, Perpina M, Greses JV. Cell type accuracy of bronchial biopsy spec imens in primary lung cancer. Chest. 1996;109:1199-203.

26. Haukoos JS, Lewis RJ. The propensity score. JAMA. 2015;314:1637-8.

27. Boffa DJ, Kosinski AS, Paul S, Mitchell JD, Onaitis M. Lymph node evaluation by open or video-assisted approaches in 11,500 anatomic lung cancer resections. Ann Thorac Surg. 2012;94:347-53.

28. Arriagada R, Bergman B, Dunant A, Le Chevalier T, Pignon J-P, Vansteenkiste J, et al. Cisplatin-based adjuvant chemotherapy in patients with completely resected non-small-cell lung cancer. N Engl J Med. 2004;350:351-60.

29. Pignon J-P, Tribodet H, Scagliotti GV, Douillard J-Y, Shepherd FA, Stephens RJ, et al. Lung adjuvant cisplatin evaluation: a pooled analysis by the LACE Collaborative Group. J Clin Oncol. 2008;26:3552-9.

30. Hancock JG, Rosen JE, Antonicelli A, Moreno A, Kim AW, Detterbeck FC, et al Impact of adjuvant treatment for microscopic residual disease after non-small cell lung cancer surgery. Ann Thorac Surg. 2015;99:406-13.

31. Strauss GM, Herndon JE, Maddaus MA, Johnstone DW, Johnson EA, Harpole DH, et al. Adjuvant paclitaxel plus carboplatin compared with observation in stage IB non-small-cell lung cancer: CALGB 9633 with the Cancer and Leukemia Group B, Radiation Therapy Oncology Group, and North Central Can cer Treatment Group Study Groups. J Clin Oncol. 2008;26:5043-51.

32. Zheng X, Schipper M, Kidwell K, Lin J, Reddy R, Ren Y, et al. Survival outcome after stereotactic body radiation therapy and surgery for stage I non-small cell lung cancer: a meta-analysis. Int J Radiat Oncol Biol Phys. 2014;90:603-11.

33. Timmerman RD, Paulus R, Pass HI. RTOG 0618: Stereotactic body radiation therapy (SBRT) to treat operable early-stage lung cancer patients. J Clin Oncol. 2013;21:suppl abstr 7523

34. Douillard J-Y, Tribodet H, Aubert D, Shepherd FA, Rosell R, Ding K, et al. Adjuvant cisplatin and vinorelbine for completely resected non-small cell lung cancer: subgroup analysis of the Lung Adjuvant Cisplatin Evaluation. J Thorac Oncol. 2010;5:220-8

35. Silvestri GA, Gonzalez AV, Jantz MA, Margolis ML, Gould MK, Tanoue LT, et al. Methods for Staging Non-small Cell Lung Cancer Diagnosis and Management of Lung Cancer, 3rd ed: American College of Chest Physicians Evidence Based Clinical Practice Guidelines. Chest. 2013;143:E211-50.

Key Words: lung cancer, stereotactic body radiotherapy, SBRT, lobectomy, survival, NSCLC 


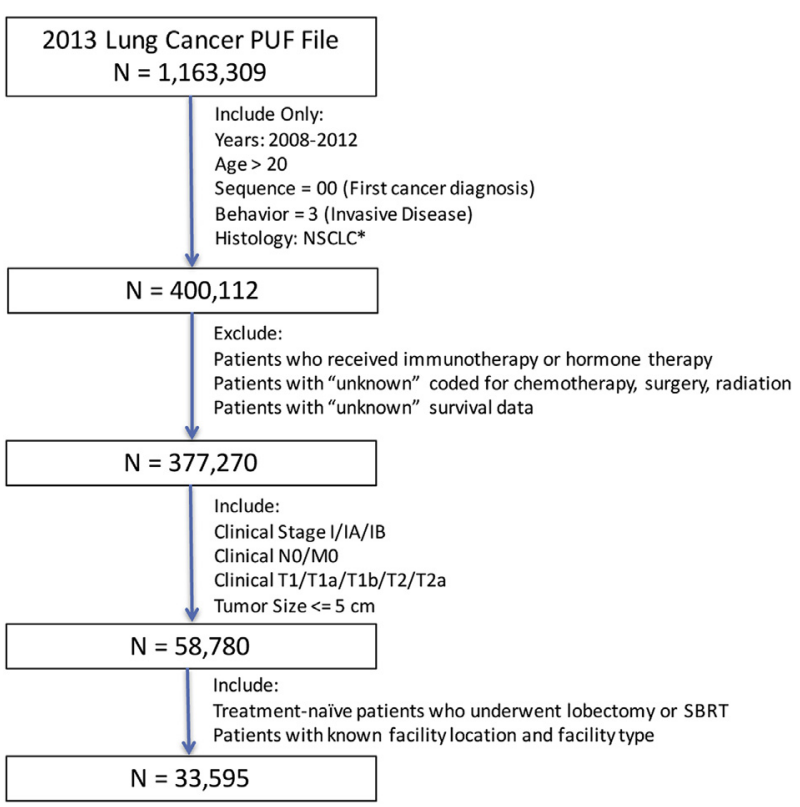

FIGURE E1. Full consort diagram detailing cohort selection. *NSCLC histology codes included 8010, 8012-8013, 8020, 8046, 8050-8052, 8070-8078, 8140-8141, 8143, 8147, 8260, 8310, 8430, 8480-8481, 8490, 8560, and 8570-8575. PUF, Participant user file; NSCLC, non-small cell lung cancer; $S B R T$, stereotactic body radiation therapy. 


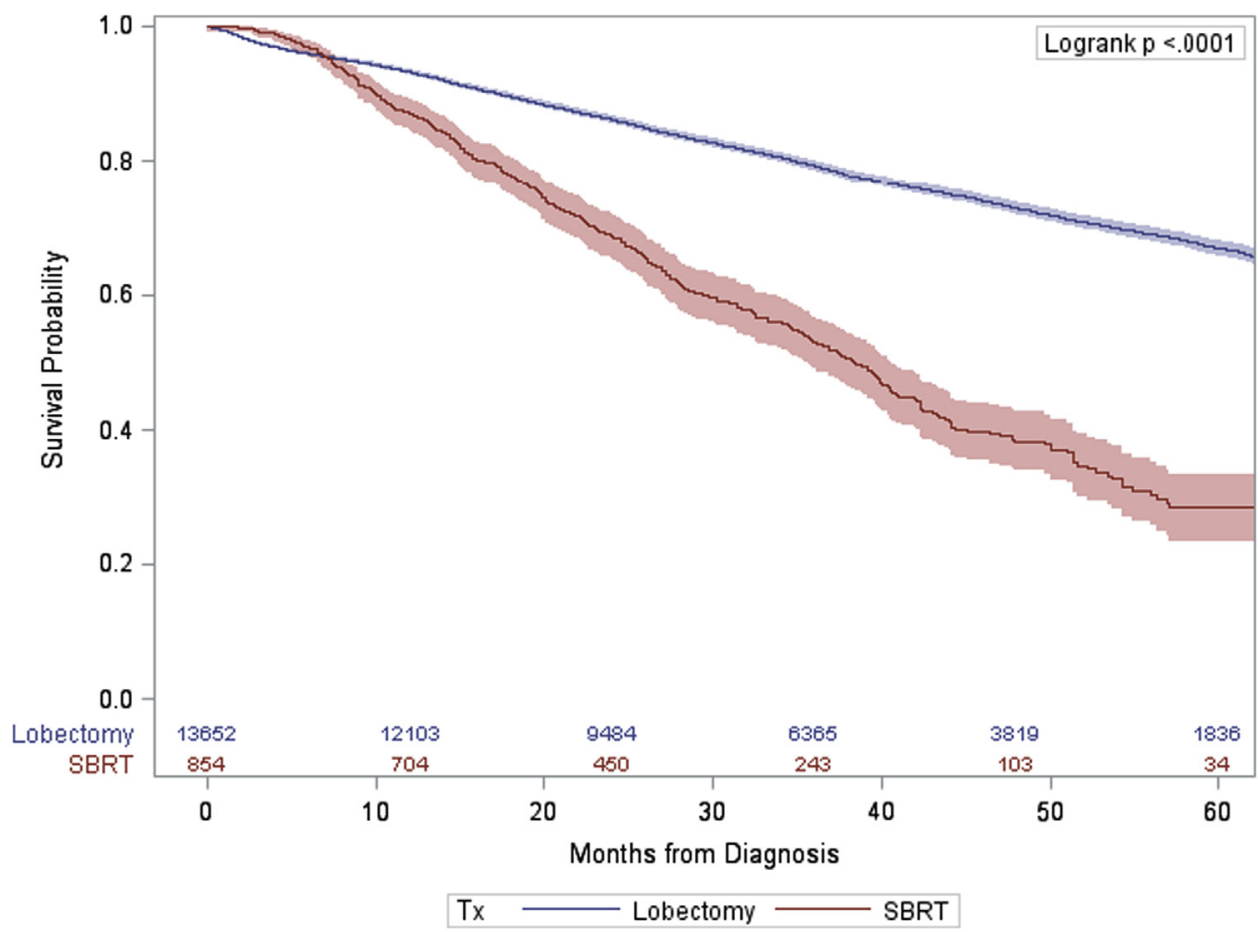

FIGURE E2. Kaplan-Meier survival analysis of the 13,652 patients who received lobectomy versus the 854 patients who received $\mathrm{SBRT}$ with a BED 10 of at least 130 Gy. SBRT, Stereotactic body radiation therapy; $T x$, treatment.

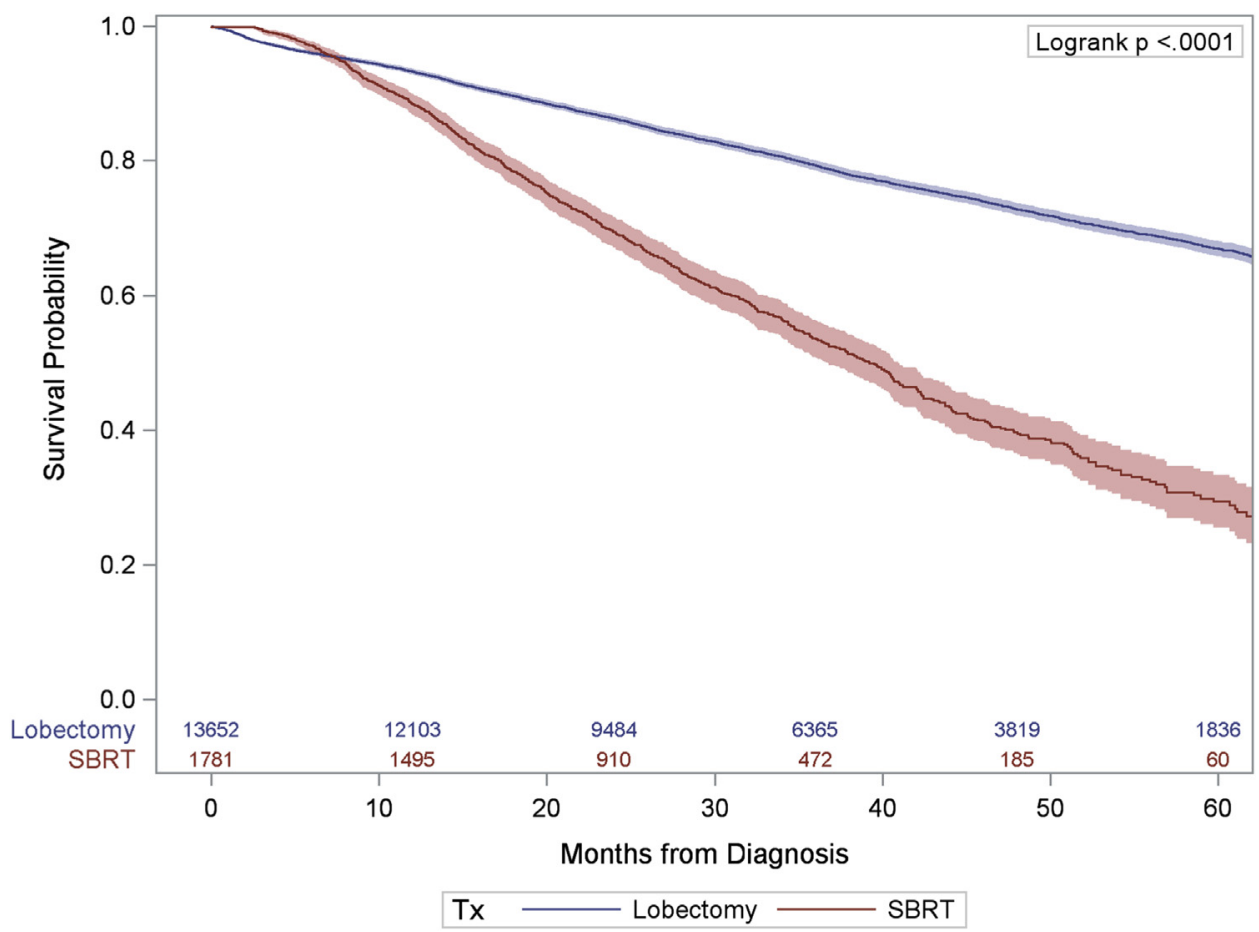

FIGURE E3. Kaplan-Meier survival analysis of the unmatched 13,652 health patients with lobectomy versus the 1781 healthy patients with SBRT. SBRT, Stereotactic body radiation therapy; $T x$, treatment. 


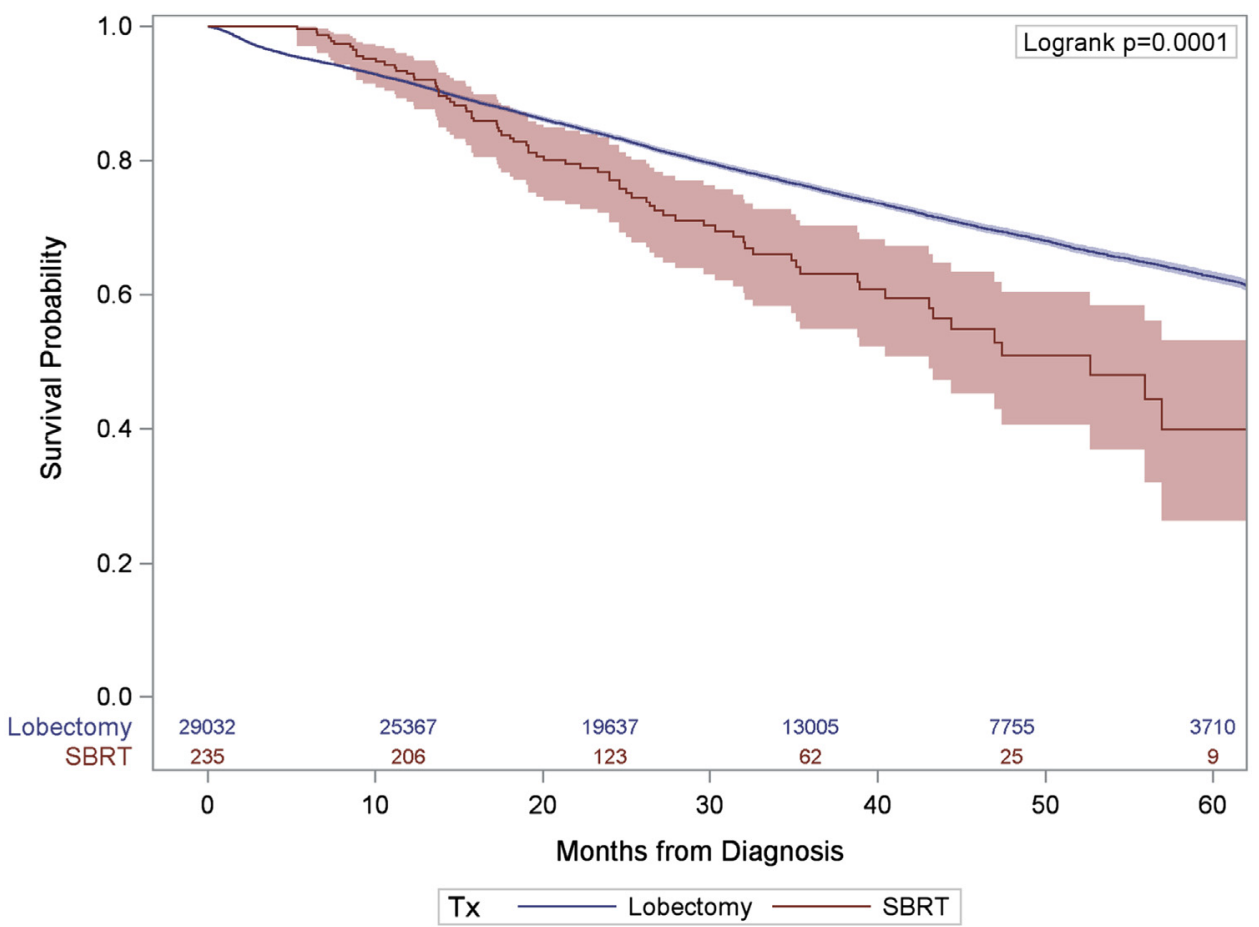

FIGURE E4. Kaplan-Meier survival analysis of the unmatched 29,032 patients with lobectomy versus the 235 patients with SBRT who were offered surgery but refused. SBRT, Stereotactic body radiation therapy; $T x$, treatment. 
TABLE E1. Cox proportional hazards model of 10,235 patients with clinical $\mathrm{T} 1$ who received lobectomy or stereotactic body radiation therapy with a minimum $\mathrm{BED}_{10}$ of $130 \mathrm{~Gy}$

\begin{tabular}{|c|c|c|c|c|}
\hline Covariate & Level & $\mathbf{N}$ & HR (LCL, UCL) & $P$ value \\
\hline \multirow[t]{2}{*}{ Treatment effect before $7.5 \mathrm{mo}$} & SBRT & 692 & Reference & \\
\hline & Lobectomy & 9543 & $1.00(0.70,1.42)$ & .99 \\
\hline \multirow[t]{2}{*}{ Treatment effect after $7.5 \mathrm{mo}$} & SBRT & 692 & Reference & \\
\hline & Lobectomy & 9543 & $0.35(0.30,0.41)$ & $<.001$ \\
\hline \multirow[t]{5}{*}{ Year of diagnosis } & 2008 & 1682 & Reference & \\
\hline & 2009 & 1955 & $1.01(0.90,1.14)$ & .86 \\
\hline & 2010 & 2073 & $0.82(0.73,0.94)$ & .003 \\
\hline & 2011 & 2204 & $0.93(0.81,1.07)$ & .30 \\
\hline & 2012 & 2321 & $0.89(0.76,1.04)$ & .13 \\
\hline \multirow[t]{2}{*}{ Facility type } & Academic & 3451 & Reference & \\
\hline & Nonacademic & 6784 & $1.23(1.13,1.35)$ & $<.001$ \\
\hline \multirow[t]{2}{*}{ Sex } & $\mathrm{F}$ & 5854 & Reference & \\
\hline & M & 4381 & $1.48(1.36,1.60)$ & $<.001$ \\
\hline Age & & & $1.02(1.02,1.03)$ & $<.001$ \\
\hline \multirow[t]{3}{*}{ Spanish/Hispanic origin } & Not Spanish/Hispanic & 9414 & Reference & \\
\hline & Spanish/Hispanic & 252 & $0.70(0.48,0.93)$ & .015 \\
\hline & Unknown & 569 & $0.95(0.79,1.14)$ & .568 \\
\hline \multirow[t]{6}{*}{ Primary payer } & Private insurance & 3489 & Reference & \\
\hline & Medicaid & 418 & $1.64(2.47,2.30)$ & $<.001$ \\
\hline & Medicare & 5839 & $1.22(1.54,1.56)$ & $<.001$ \\
\hline & No insurance & 220 & $1.08(2.07,2.59)$ & .016 \\
\hline & Other government & 124 & $0.78(1.73,2.08)$ & .448 \\
\hline & Unknown & 145 & $1.10(2.24,1.86)$ & .012 \\
\hline \multirow{6}{*}{ Primary site } & Lower lobe & 3136 & Reference & \\
\hline & Lung, NOS & 144 & $1.23(0.90,1.68)$ & .20 \\
\hline & Main bronchus & 17 & $1.07(0.10,11.80)$ & .96 \\
\hline & Middle lobe & 571 & $1.01(0.83,1.22)$ & .92 \\
\hline & Overlapping lesion & 43 & $2.17(1.32,3.57)$ & .0023 \\
\hline & Upper lobe & 6324 & $0.99(0.90,1.09)$ & .85 \\
\hline \multirow[t]{3}{*}{ Laterality } & Left & 4165 & Reference & \\
\hline & Other* & 22 & $0.66(0.09,4.70)$ & .678 \\
\hline & Right & 6048 & $1.05(0.97,1.15)$ & .22 \\
\hline \multirow[t]{4}{*}{ Histology } & Adenocarcinoma & 7126 & Reference & \\
\hline & Large cell carcinoma & 216 & $1.40(1.06,1.85)$ & .018 \\
\hline & Other NSCLC & 430 & $1.25(1.04,1.49)$ & .015 \\
\hline & Squamous cell carcinoma & 2463 & $1.14(1.04,1.25)$ & .007 \\
\hline \multirow[t]{5}{*}{ Grade } & 1 & 1570 & Reference & \\
\hline & 2 & 4863 & $1.59(1.36,1.86)$ & $<.001$ \\
\hline & 3 & 2936 & $1.75(1.49,2.06)$ & $<.001$ \\
\hline & 4 & 109 & $1.45(0.94,2.24)$ & .091 \\
\hline & Unknown & 757 & $1.68(1.36,2.08)$ & $<.001$ \\
\hline Tumor size & & & $1.02(1.02,1.03)$ & $<.001$ \\
\hline
\end{tabular}


TABLE E2. Cox proportional hazards model of 4271 patients with clinical T2 who received lobectomy or stereotactic body radiation therapy with a minimum BED $\mathrm{BD}_{10}$ of $130 \mathrm{~Gy}$

\begin{tabular}{|c|c|c|c|c|}
\hline Covariate & Level & $\mathbf{N}$ & HR (LCL, UCL) & $P$ value \\
\hline \multirow[t]{2}{*}{ Treatment effect before $7.5 \mathrm{mo}$} & SBRT & 162 & Reference & \\
\hline & Lobectomy & 4109 & $1.03(0.56,1.91)$ & .92 \\
\hline \multirow[t]{2}{*}{ Treatment effect after $7.5 \mathrm{mo}$} & SBRT & 162 & Reference & \\
\hline & Lobectomy & 4109 & $0.41(0.32,0.53)$ & $<.001$ \\
\hline \multirow[t]{5}{*}{ Year of diagnosis } & 2008 & 797 & Reference & \\
\hline & 2009 & 954 & $0.99(0.86,1.15)$ & .93 \\
\hline & 2010 & 812 & $0.96(0.81,1.14)$ & .64 \\
\hline & 2011 & 837 & $0.92(0.77,1.11)$ & .40 \\
\hline & 2012 & 871 & $0.84(0.68,1.05)$ & .12 \\
\hline \multirow[t]{2}{*}{ Facility type } & Academic & 1386 & Reference & \\
\hline & Nonacademic & 2885 & $1.26(1.11,1.42)$ & $<.001$ \\
\hline \multirow[t]{2}{*}{ Sex } & $\mathrm{F}$ & 2180 & Reference & \\
\hline & M & 2091 & $1.38(1.24,1.55)$ & $<.001$ \\
\hline Age & & & $1.03(1.02,1.03)$ & $<.001$ \\
\hline \multirow[t]{6}{*}{ Primary payer } & Private insurance & 1401 & Reference & \\
\hline & Medicaid & 195 & $1.40(1.06,1.85)$ & .017 \\
\hline & Medicare & 2463 & $1.23(1.06,1.42)$ & .007 \\
\hline & No insurance & 106 & $0.70(0.43,1.15)$ & .16 \\
\hline & Other government & 46 & $1.04(0.60,1.79)$ & .89 \\
\hline & Unknown & 60 & $0.97(0.57,1.63)$ & .90 \\
\hline \multirow[t]{3}{*}{ Median income } & $<\$ 48,000$ & 1818 & Reference & \\
\hline & $\$ 48,000+$ & 2394 & $1.01(0.90,1.14)$ & .84 \\
\hline & Unknown & 59 & $2.21(1.43,3.42)$ & $<.001$ \\
\hline \multirow[t]{4}{*}{ Urban/rural } & Rural & 73 & Reference & \\
\hline & Metro & 3401 & $1.05(0.69,1.62)$ & .81 \\
\hline & Unknown & 131 & $1.93(1.15,3.24)$ & .013 \\
\hline & Urban & 666 & $1.23(0.80,1.92)$ & .34 \\
\hline \multirow[t]{6}{*}{ Primary site } & Lower lobe & 1447 & Reference & \\
\hline & Lung, NOS & 63 & $1.04(0.68,1.58)$ & .86 \\
\hline & Main bronchus & $\sim$ & $\sim$ & $\sim$ \\
\hline & Middle lobe & 205 & $1.01(0.77,1.32)$ & .93 \\
\hline & Overlapping lesion & 45 & $1.19(0.73,1.94)$ & .48 \\
\hline & Upper lobe & 2507 & $0.91(0.81,1.03)$ & .12 \\
\hline \multirow[t]{3}{*}{ Laterality } & Left & 1722 & Reference & \\
\hline & Other* & $\sim$ & $2.49(0.62,10.10)$ & .20 \\
\hline & Right & 2541 & $0.96(0.86,1.08)$ & .53 \\
\hline \multirow[t]{4}{*}{ Histology } & Adenocarcinoma & 2639 & Reference & \\
\hline & Large cell carcinoma & 107 & $1.92(1.38,2.69)$ & $<.001$ \\
\hline & Other NSCLC & 187 & $1.29(1.01,1.65)$ & .045 \\
\hline & Squamous cell carcinoma & 1338 & $1.16(1.03,1.31)$ & .015 \\
\hline \multirow[t]{5}{*}{ Grade } & 1 & 423 & Reference & \\
\hline & 2 & 1917 & $1.70(1.34,2.16)$ & $<.001$ \\
\hline & 3 & 1616 & $1.83(1.43,2.33)$ & $<.001$ \\
\hline & 4 & 71 & $0.92(0.53,1.58)$ & .76 \\
\hline & Unknown & 244 & $1.46(1.05,2.02)$ & .022 \\
\hline Tumor size & & & $1.01(1.01,1.02)$ & $<.001$ \\
\hline
\end{tabular}

Note that per NCDB requirements, some values are censored with a " " due to low n values. $H R$, Hazard ratio; $L C L$, lower confidence limit; $U C L$, upper confidence limit; $S B R T$, stereotactic body radiation therapy; $F$, female; $M$, male; NOS, not otherwise specified; $N S C L C$, non-small cell lung cancer. *Other laterality designates main bronchus and other central tumors. 
TABLE E3. Bivariate analysis of lobectomy and operable patients with stereotactic body radiation therapy who were coded as being offered surgery but refused it before and after propensity score matching

\begin{tabular}{|c|c|c|c|c|c|c|}
\hline & \multicolumn{3}{|c|}{ Unmatched cohort } & \multicolumn{3}{|c|}{ Matched cohort } \\
\hline & $\begin{array}{c}\text { Lobectomy } \\
(\mathrm{N}=\mathbf{2 9 , 0 3 2}) \\
\end{array}$ & $\begin{array}{c}\text { SBRT } \\
(\mathbf{N}=\mathbf{2 3 5}) \\
\end{array}$ & & $\begin{array}{c}\begin{array}{c}\text { Lobectomy } \\
(N=235)\end{array} \\
\end{array}$ & $\begin{array}{c}\text { SBRT } \\
(\mathbf{N}=\mathbf{2 3 5}) \\
\end{array}$ & \\
\hline & $\mathbf{N}(\%)$ & $\mathbf{N}(\%)$ & $P$ value & $\mathbf{N}(\%)$ & $\mathbf{N}(\%)$ & $P$ value \\
\hline Year of diagnosis & & & $<.001$ & & & .91 \\
\hline 2008 & $5158(18)$ & $13(6)$ & & $15(6)$ & $13(6)$ & \\
\hline 2009 & $5762(20)$ & $39(17)$ & & $41(17)$ & $39(17)$ & \\
\hline 2010 & $5757(20)$ & $41(17)$ & & $47(20)$ & $41(17)$ & \\
\hline 2011 & $5999(21)$ & $59(25)$ & & $55(23)$ & $59(25)$ & \\
\hline 2012 & $6356(22)$ & $83(35)$ & & $77(33)$ & $83(35)$ & \\
\hline Sex & & & .037 & & & .3 \\
\hline F & $15,319(53)$ & $140(60)$ & & $151(64)$ & $140(60)$ & \\
\hline M & $13,713(47)$ & $95(40)$ & & $84(36)$ & $95(40)$ & \\
\hline \multicolumn{7}{|l|}{ Age (y) } \\
\hline Mean (SD) & $66.9(9.7)$ & $75.3(8.9)$ & $<.001$ & $75.0(8.2)$ & $75.3(8.9)$ & .71 \\
\hline Race & & & .84 & & & .55 \\
\hline Nonwhite & $3459(12)$ & $27(11)$ & & $23(10)$ & $27(11)$ & \\
\hline White & $25,573(88)$ & $208(89)$ & & $212(90)$ & $208(89)$ & \\
\hline Spanish Hispanic origin & & & .17 & & & .89 \\
\hline Not Spanish/Hispanic & $26,579(92)$ & 209 (89) & & $206(88)$ & 209 (89) & \\
\hline Spanish/Hispanic origin & $702(2)$ & $\sim$ & & $\sim$ & $\sim$ & \\
\hline Unknown & $1751(6)$ & $21(9)$ & & $24(10)$ & $21(9)$ & \\
\hline Primary payer & & & $<.001$ & & & .99 \\
\hline Medicaid & $1391(5)$ & $\sim$ & & $\sim$ & $\sim$ & \\
\hline Medicare & $17,343(60)$ & $190(81)$ & & $190(81)$ & $190(81)$ & \\
\hline No insurance & $647(2)$ & $\sim$ & & $\sim$ & $\sim$ & \\
\hline Other government & $314(1)$ & $\sim$ & & $\sim$ & $\sim$ & \\
\hline Private Insurance/managed care & $9013(31)$ & $28(12)$ & & $31(13)$ & $28(12)$ & \\
\hline Unknown & $324(1)$ & $\sim$ & & $\sim$ & $\sim$ & \\
\hline Median income & & & .42 & & & .6 \\
\hline$\$ 48,000+$ & $15,846(55)$ & $135(57)$ & & $135(57)$ & $135(57)$ & \\
\hline$<\$ 48,000$ & $12,847(44)$ & $99(42)$ & & $97(41)$ & $99(42)$ & \\
\hline Unknown & $339(1)$ & $\sim$ & & $\sim$ & $\sim$ & \\
\hline Percent no high school degree & & & .026 & & & .47 \\
\hline$\geq 21 \%$ & $4701(16)$ & $31(13)$ & & $26(11)$ & $31(13)$ & \\
\hline $13 \%-20.9 \%$ & $8235(28)$ & $60(26)$ & & $63(27)$ & $60(26)$ & \\
\hline $7 \%-12.9 \%$ & $9782(34)$ & $75(32)$ & & $87(37)$ & $75(32)$ & \\
\hline$<7 \%$ & $5983(21)$ & $68(29)$ & & $56(24)$ & $68(29)$ & \\
\hline Unknown & $331(1)$ & $\sim$ & & $\sim$ & $\sim$ & \\
\hline Urban/rural 2013 & & & .21 & & & .38 \\
\hline Metro & $22,843(79)$ & $198(84)$ & & $200(85)$ & $198(84)$ & \\
\hline Rural & $642(2)$ & $\sim$ & & $\sim$ & $\sim$ & \\
\hline Unknown & $861(3)$ & $\sim$ & & $\sim$ & $\sim$ & \\
\hline Urban & $4686(16)$ & $28(12)$ & & $28(12)$ & $28(12)$ & \\
\hline Charlson- Deyo score & & & $<.001$ & & & .97 \\
\hline 0 & $13,652(47)$ & $157(67)$ & & $156(66)$ & $157(67)$ & \\
\hline 1 & $10,877(37)$ & $50(21)$ & & $52(22)$ & $50(21)$ & \\
\hline $2+$ & $4503(16)$ & $28(12)$ & & $27(11)$ & $28(12)$ & \\
\hline Facility type & & & $<.001$ & & & .78 \\
\hline Academic/research program (including NCI) & $8846(30)$ & $99(42)$ & & $96(41)$ & $99(42)$ & \\
\hline Nonacademic program & $20,186(70)$ & $136(58)$ & & $139(59)$ & $136(58)$ & \\
\hline Facility location & & & .011 & & & .84 \\
\hline Central & $13,268(46)$ & $115(49)$ & & $114(49)$ & $115(49)$ & \\
\hline Mountain & $1024(4)$ & $14(6)$ & & $\sim$ & $14(6)$ & \\
\hline
\end{tabular}




\begin{tabular}{|c|c|c|c|c|c|c|}
\hline & \multicolumn{3}{|c|}{ Unmatched cohort } & \multicolumn{3}{|c|}{ Matched cohort } \\
\hline & $\begin{array}{c}\text { Lobectomy } \\
(N=\mathbf{2 9 , 0 3 2})\end{array}$ & $\begin{array}{c}\text { SBRT } \\
(\mathbf{N}=\mathbf{2 3 5})\end{array}$ & & $\begin{array}{c}\text { Lobectomy } \\
(\mathbf{N}=\mathbf{2 3 5})\end{array}$ & $\begin{array}{c}\text { SBRT } \\
(\mathbf{N}=\mathbf{2 3 5})\end{array}$ & \\
\hline & $\mathbf{N}(\%)$ & $\mathbf{N}(\%)$ & $P$ value & $\mathbf{N}(\%)$ & $\mathbf{N}(\%)$ & $P$ value \\
\hline North East & $4882(17)$ & $48(20)$ & & $53(23)$ & $48(20)$ & \\
\hline Pacific & $2651(9)$ & $12(5)$ & & $11(5)$ & $12(5)$ & \\
\hline South Atlantic & $7207(25)$ & $46(20)$ & & $48(20)$ & $46(20)$ & \\
\hline Primary site & & & .18 & & & .84 \\
\hline Lower lobe & $9022(31)$ & $62(26)$ & & $57(24)$ & $62(26)$ & \\
\hline Lung, NOS & $335(1)$ & $\sim$ & & $\sim$ & $\sim$ & \\
\hline Main bronchus & $48(0)$ & $\sim$ & & $\sim$ & $\sim$ & \\
\hline Middle lobe & $1560(5)$ & $\sim$ & & $\sim$ & $\sim$ & \\
\hline Overlapping lesion & $180(1)$ & $\sim$ & & $\sim$ & $\sim$ & \\
\hline Upper lobe & $17,887(62)$ & $161(69)$ & & $169(72)$ & $161(69)$ & \\
\hline Laterality & & & .31 & & & .85 \\
\hline Left & $11,678(40)$ & $105(45)$ & & $107(46)$ & $105(45)$ & \\
\hline Other* & $64(0)$ & $0(0)$ & & & & \\
\hline Right & $17,290(60)$ & $130(55)$ & & 128 (54) & $130(55)$ & \\
\hline Histology & & & $<.001$ & & & .66 \\
\hline Adenocarcinoma & $18,200(63)$ & $125(53)$ & & $125(53)$ & $125(53)$ & \\
\hline Large cell carcinoma & 797 (3) & $\sim$ & & $\sim$ & $\sim$ & \\
\hline Other NSCLC & $905(3)$ & $39(17)$ & & $45(19)$ & $39(17)$ & \\
\hline Squamous cell carcinoma & $9130(31)$ & $69(29)$ & & $61(26)$ & $69(29)$ & \\
\hline Grade & & & $<.001$ & & & $<.001$ \\
\hline 1 & $3570(12)$ & $16(7)$ & & $33(14)$ & $16(7)$ & \\
\hline 2 & $14,048(48)$ & $45(19)$ & & $96(41)$ & $45(19)$ & \\
\hline 3 & $10,023(35)$ & $40(17)$ & & $89(38)$ & $40(17)$ & \\
\hline 4 & $380(1)$ & $\sim$ & & $\sim$ & $\sim$ & \\
\hline Unknown & $1011(3)$ & $133(57)$ & & $14(6)$ & $133(57)$ & \\
\hline Tumor size $(\mathrm{mm})$ & & & .097 & & & .38 \\
\hline Mean (SD) & $25.1(10.5)$ & $24.0(9.3)$ & & $23.6(9.9)$ & $24.0(9.3)$ & \\
\hline Clinical T stage & & & .28 & & & .91 \\
\hline 1 & $20,555(71)$ & $174(74)$ & & 175 (74) & 174 (74) & \\
\hline 2 & 8477 (29) & $61(26)$ & & $60(26)$ & $61(26)$ & \\
\hline \multicolumn{7}{|l|}{ 30-d mortality } \\
\hline No & $27,906(96)$ & & & $224(95)$ & & \\
\hline Yes & $667(2)$ & & & $\sim$ & & \\
\hline Unknown & $459(2)$ & & & $\sim$ & & \\
\hline \multicolumn{7}{|l|}{ 90-d mortality } \\
\hline No & $27,205(94)$ & & & $212(90)$ & & \\
\hline Yes & $1163(4)$ & & & $14(6)$ & & \\
\hline Unknown & $664(2)$ & & & $\sim$ & & \\
\hline
\end{tabular}


TABLE E4. Cox proportional hazards model of 29,267 patients who underwent lobectomy or received stereotactic body radiation therapy after refusing surgery

\begin{tabular}{|c|c|c|c|c|}
\hline Covariate & Level & $\mathbf{N}$ & HR (LCL, UCL) & $P$ value \\
\hline \multirow[t]{2}{*}{ Treatment effect before $15 \mathrm{mo}^{*}$} & SBRT & 235 & Reference & \\
\hline & Lobectomy & 29,032 & $1.06(0.71,1.57)$ & .78 \\
\hline \multirow[t]{2}{*}{ Treatment effect after $15 \mathrm{mo}$} & SBRT & 235 & Reference & \\
\hline & Lobectomy & 29,032 & $0.59(0.45,0.79)$ & $<.001$ \\
\hline \multirow[t]{5}{*}{ Year of diagnosis } & 2008 & 5171 & Reference & \\
\hline & 2009 & 5801 & $0.99(0.93,1.06)$ & .82 \\
\hline & 2010 & 5798 & $0.94(0.88,1.01)$ & .094 \\
\hline & 2011 & 6058 & $0.90(0.83,0.97)$ & .0048 \\
\hline & 2012 & 6439 & $0.88(0.80,0.95)$ & .0022 \\
\hline \multirow[t]{2}{*}{ Facility type } & Academic & 8945 & Reference & \\
\hline & Nonacademic & 20,322 & $1.13(1.07,1.19)$ & $<.001$ \\
\hline \multirow[t]{5}{*}{ Facility location } & North East & 4930 & Reference & \\
\hline & Central & 13,383 & $1.134(1.06,1.22)$ & $<.001$ \\
\hline & Mountain & 1038 & $1.04(0.91,1.19)$ & .58 \\
\hline & Pacific & 2663 & $1.09(1.00,1.20)$ & .062 \\
\hline & South Atlantic & 7253 & $1.11(1.03,1.19)$ & .0078 \\
\hline \multirow[t]{2}{*}{ Sex } & $\mathrm{F}$ & 15,459 & Reference & \\
\hline & M & 13,808 & $1.33(1.27,1.39)$ & $<.001$ \\
\hline Age & & & $1.03(1.03,1.03)$ & $<.001$ \\
\hline \multirow[t]{3}{*}{ Spanish/Hispanic origin } & Not Spanish/Hispanic & 26,788 & Reference & \\
\hline & Spanish/Hispanic & 707 & $0.78(0.66,0.92)$ & .0029 \\
\hline & Unknown & 1772 & $1.02(0.93,1.12)$ & .62 \\
\hline \multirow[t]{4}{*}{ Urban/rural } & Rural & 645 & Reference & \\
\hline & Metro & 23,041 & $1.04(0.89,1.21)$ & .63 \\
\hline & Unknown & 867 & $1.23(1.00,1.51)$ & .053 \\
\hline & Urban & 4714 & $1.15(0.98,1.34)$ & .086 \\
\hline \multirow[t]{5}{*}{ Percent no high school diploma } & $\geq 21 \%$ & 4732 & Reference & \\
\hline & $13 \%-20.9 \%$ & 8295 & $0.99(0.93,1.06)$ & .81 \\
\hline & $7 \%-12.9 \%$ & 9857 & $0.94(0.88,1.01)$ & .078 \\
\hline & $<7 \%$ & 6051 & $0.89(0.83,0.97)$ & .0051 \\
\hline & Unknown & 332 & $3.09(2.51,3.80)$ & $<.001$ \\
\hline \multirow[t]{6}{*}{ Insurance status } & Private insurance & 9041 & Reference & \\
\hline & Medicaid & 1400 & $1.53(1.37,1.70)$ & $<.001$ \\
\hline & Medicare & 17,533 & $1.14(1.07,1.21)$ & $<.001$ \\
\hline & No insurance & 650 & $1.15(0.97,1.38)$ & .11 \\
\hline & Other government & 318 & $1.12(0.90,1.41)$ & .31 \\
\hline & Unknown & 325 & $1.23(0.98,1.54)$ & .068 \\
\hline \multirow[t]{3}{*}{ Charlson score } & 0 & 13,809 & Reference & \\
\hline & 1 & 10,927 & $1.17(1.11,1.23)$ & $<.001$ \\
\hline & $2+$ & 4531 & $1.47(1.38,1.56)$ & $<.001$ \\
\hline \multirow[t]{6}{*}{ Primary site } & Lower lobe & 9084 & Reference & \\
\hline & Lung, NOS & 339 & $1.05(0.85,1.29)$ & .67 \\
\hline & Main bronchus & 48 & $0.71(0.26,1.96)$ & .51 \\
\hline & Middle lobe & 1568 & $0.96(0.87,1.07)$ & .48 \\
\hline & Overlapping lesion & 180 & $1.23(0.96,1.59)$ & .10 \\
\hline & Upper lobe & 18,048 & $0.93(0.89,0.98)$ & .0044 \\
\hline \multirow[t]{3}{*}{ Laterality } & Left & 11,783 & Reference & \\
\hline & Other $\dagger$ & 64 & $1.18(0.53,2.62)$ & .69 \\
\hline & Right & 17,420 & $1.03(0.99,1.08)$ & .16 \\
\hline \multirow[t]{4}{*}{ Histology } & Adenocarcinoma & 18,325 & Reference & \\
\hline & Large cell carcinoma & 799 & $1.29(1.13,1.47)$ & $<.001$ \\
\hline & Other NSCLC & 944 & $1.09(0.97,1.23)$ & .15 \\
\hline & Squamous cell carcinoma & 9199 & $1.03(0.98,1.08)$ & .19 \\
\hline
\end{tabular}


TABLE E4. Continued

\begin{tabular}{|c|c|c|c|c|}
\hline Covariate & Level & $\mathbf{N}$ & HR (LCL, UCL) & $P$ value \\
\hline \multirow[t]{5}{*}{ Grade } & 1 & 3586 & Reference & \\
\hline & 2 & 14,093 & $1.55(1.41,1.69)$ & $<.001$ \\
\hline & 3 & 10,063 & $1.82(1.66,2.00)$ & $<.001$ \\
\hline & 4 & 381 & $1.49(1.20,1.85)$ & $<.001$ \\
\hline & Unknown & 1144 & $1.67(1.45,1.92)$ & $<.001$ \\
\hline Tumor size & & & $1.02(1.01,1.02)$ & $<.001$ \\
\hline \multirow[t]{2}{*}{ Clinical T stage } & 1 & 20,729 & Reference & \\
\hline & 2 & 8538 & $1.02(0.96,1.08)$ & .56 \\
\hline
\end{tabular}

HR, Hazard ratio; $L C L$, lower confidence limit; $U C L$, upper confidence limit; $S B R T$, stereotactic body radiation therapy; $F$, female; $M$, male; NOS, not otherwise specified; NSCLC, non-small cell lung cancer. *Note that as seen in Figure E4, the point where the SBRT and lobectomy curves cross is pushed out to 15 months in this subset analysis; therefore, the Cox analyses were stratified at this time point. †Other laterality designates main bronchus and other central tumors. 